\title{
Aves do Parque Estadual Carlos Botelho - SP
}

\author{
Alexsander Zamorano Antunes ${ }^{1,4}$, Bruna Gonçalves da Silva ${ }^{2}$, Ciro Koiti Matsukuma ${ }^{1}$, \\ Marilda Rapp de Eston ${ }^{1}$ \& Ana Maria Rodrigues dos Santos ${ }^{3}$ \\ IInstituto Florestal, Rua do Horto, 931, CEP 02377-000, São Paulo, SP, Brasil \\ ${ }^{2}$ Programa de Pós-graduação em Ecologia, Instituto de Biologia, Universidade Estadual de \\ Campinas - UNICAMP, Av. Bertrand Russel, s/n, Cidade Universitária Zeferino Vaz, \\ Barão Geraldo, CEP 13083-970, Campinas, SP, Brasil \\ ${ }^{3}$ Escola Estadual Professora Rita Bicudo Pereira, Av. Peri Ronchetti, 494, \\ Jardim Peri, CEP 02633-000, São Paulo, SP, Brasil \\ ${ }^{4}$ Autor para correspondência: Alexsander Zamorano Antunes, e-mail: alexza@if.sp.gov.br
}

ANTUNES, A.Z., SILVA, B.G., MATSUKUMA, C.K., ESTON, M.R. \& SANTOS, A.M.R. Birds from Carlos Botelho Park state of São Paulo southeastern Brazil. Biota Neotrop. 13(2): http://www.biotaneotropica.org. br/v13n2/en/abstract?inventory+bn00513022013

Abstract: The biodiversity inventory in protected areas from Atlantic Forest can increase knowledge about species distribution and contribute to monitoring and management actions that enhance the ecosystems conservation. Our objectives were to characterize bird assemblages at Carlos Botelho State Park, São Paulo southeastern Brazil, regarding species composition, relative abundance and association with local vegetation types. Sampling was conducted between April 2006 and December 2009 by transects, resulting in 1,000 hours of sampling effort. We recorded 331 species, most showed relative abundance lower than 100 detections. Twenty-five species are threatened in the state of São Paulo.

Keywords: conservation, Atlantic Forest, inventory.

ANTUNES, A.Z., SILVA, B. G., MATSUKUMA, C.K., ESTON, M.R. \& SANTOS, A.M.R. Aves do Parque Estadual Carlos Botelho - SP. Biota Neotrop. 13(2): http://www.biotaneotropica.org.br/v13n2/pt/abstract?inv entory+bn00513022013

Resumo: O inventário da biodiversidade nas unidades de proteção integral implantadas em remanescentes da Mata Atlântica, além de ampliar o conhecimento sobre a distribuição das espécies, pode contribuir para ações de monitoramento e manejo que aprimorem a conservação dos ecossistemas. Os objetivos desse trabalho foram caracterizar as assembleias de aves do Parque Estadual Carlos Botelho, sudeste do estado de São Paulo, quanto à composição de espécies, abundância relativa e associação com fitofisionomias locais. A amostragem foi realizada entre abril de 2006 e dezembro de 2009 pelo método de trajetos de distância ilimitada, totalizando 1000 horas de esforço amostral. Foram registradas 331 espécies, predominando formas florestais residentes e com abundância relativa inferior a 100 detecções. Vinte e cinco espécies estão ameaçadas de extinção no estado de São Paulo.

Palavras-chave: conservação, Mata Atlântica, inventário. 


\section{Introdução}

Apesar de atualmente serem amplamente reconhecidos pela sua importância biológica e para a manutenção dos chamados serviços ambientais, os ecossistemas integrantes da Mata Atlântica continuam a ser reduzidos e degradados, subsistindo em pouco mais de $12 \%$ da sua área de distribuição original (Mittermeier et al. 2004, Ribeiro et al. 2011). O estabelecimento e manejo adequado de unidades de conservação de proteção integral - UPIs, se impõem como a maneira mais efetiva de resguardar a sua biodiversidade no curto prazo. Recentemente, baseando-se no conhecimento disponível sobre diversos grupos biológicos, foram indicadas áreas relevantes para o estabelecimento de tais unidades (Brasil 2007, Rodrigues \& Bononi 2008). Além disso, como apontado por Terborgh \& Van Schaik (2002), as UPIs existentes necessitam ser adequadamente protegidas de vetores de degradação ilegais (ex. caça, corte seletivo, etc.) e legais (ex. estabelecimento de rodovias, linhas de transmissão de energia elétrica, gasodutos, etc). Muitas UPIs apresentam deficiências de concepção que contribuem para intensificar estes impactos, tais como tamanho insuficiente para manter populações viáveis da maior parte das espécies no longo prazo, sobreposição com áreas tradicionalmente ocupadas por populações humanas, etc (Terborgh \& Van Schaik, 2002, Ribeiro et al. 2011).

O Maciço de Paranapiacaba no sudeste do estado de São Paulo engloba um conjunto de cinco UPIs, Parques Estaduais Carlos Botelho, Intervales, Nascentes do Paranapanema e Turístico do Alto Ribeira (PETAR) e Estação Ecológica de Xitué, totalizando aproximadamente 140.000 ha, que tanto em relação aos aspectos de métricas da paisagem quanto pelos resultados disponíveis de caracterização da sua biodiversidade, constituem-se no remanescente mais representativo da Floresta Ombrófila Densa da Mata Atlântica (Bencke et al. 2006, Galetti et al. 2009 , Ribeiro et al. 2011). Considerando também, que as unidades do maciço apresentam históricos e intensidades de uso antrópico distintos, e que os vetores de pressão sobre as UPIs podem variar com o tempo, a caracterização da biota, visando colaborar com programas de monitoramento das condições ambientais locais, é de grande relevância. As aves podem ser incluídas nesses programas por serem bem conhecidas e relativamente fáceis de amostrar, quando comparadas a outros grupos de animais. Adicionalmente, listas locais acuradas podem contribuir para ampliar o conhecimento sobre biogeografia e ecologia de comunidades de aves (Cohn-Haft et al. 1997). Das unidades do Maciço, Intervales apresenta a avifauna melhor caracterizada, com dados de riqueza e abundância relativa obtidos em estudos de longo prazo (Vielliard \& Silva 2001, Aleixo \& Galetti 1997).

Visando aprimorar o conhecimento sobre a avifauna do Maciço, os objetivos do presente estudo foram caracterizar assembleias de aves do Parque Estadual Carlos Botelho, quanto à composição de espécies, abundância relativa e associação com fitofisionomias locais.

\section{Material e Métodos}

\section{1. Área de estudo}

O Parque Estadual Carlos Botelho - PECB, localizado entre as coordenadas $24^{\circ} 06^{\prime} 55^{\prime \prime}$ - $24^{\circ} 14^{\prime} 41^{\prime \prime} \mathrm{S}$ e $47^{\circ} 47^{\prime} 18^{\prime \prime}$ - $48^{\circ} 07^{\prime}$ $17^{\prime}$ 'W, engloba 37.644,36 ha, distribuídos pelos municípios paulistas de Capão Bonito, São Miguel Arcanjo, Sete Barras e Tapiraí. A amplitude altitudinal local varia de 20 a $1000 \mathrm{~m}$, incluindo trechos do Planalto de Guapiara (bacia do Alto Paranapanema), da Serra de Paranapiacaba e Vale do rio Ribeira de Iguape. O clima pode ser classificado, no sistema de Köppen, em quente úmido sem estiagem (Cfa) para as áreas submontanas e de baixada, e temperado úmido sem estiagem $(\mathrm{Cfb})$ nas áreas montanas. A temperatura média anual fica entre 18 e $20^{\circ} \mathrm{C}$ e a pluviosidade anual entre 1500 e $2200 \mathrm{~mm}$ (São Paulo, 2008a). A formação vegetal predominante é a Floresta Ombrófila Densa, sendo que neste estudo foram amostradas duas fitofisionomias (Instituto... 2012): a Floresta Submontana (entre 30-400 m de altitude) e a Floresta Montana (500-1000 m). Além dessas, foram pesquisadas formações de origem antrópica que ocupam áreas restritas no interior do parque: um reflorestamento de pinheirosdo-Paraná Araucaria angustifolia (Bertol.) Kuntze com sub-bosque de espécies nativas; gramados e jardins no entorno das edificações das sedes dos núcleos administrativos; vegetação de brejo no entorno de pequenos açudes e áreas em que predominam os estádios iniciais e intermediários da sucessão secundária (capoeiras). Na Figura 1 são apresentadas as trilhas e estradas amostradas e a distribuição das categorias fitofisionômicas reconhecidas.

O parque é uma unidade de proteção integral plenamente consolidada. Toda a sua área é de domínio público e foi regularizada há mais de 50 anos, dispõe de Plano de Manejo em implantação e a fiscalização intensiva, priorizada desde a sua criação, possibilitou a manutenção de seu território e de suas condições ambientais (São Paulo, 2008a). Essas características viabilizam o estudo de comunidades de vertebrados relativamente pouco impactadas pela ação humana (Beisiegel 2009, Galetti et al. 2009, Brocardo et al. 2012).

As pesquisas publicadas sobre a avifauna incluem levantamentos preliminares e observações sobre ecologia e comportamento para algumas espécies (Willis \& Oniki 1981, 2003, Pacagnella et al. 1994, Straube \& Scherer-Neto 1995, Galetti et al. 1997, Guix et al. 2002, Hernández et al. 2002, Pacheco \& Fonseca 2002, Pedrocchi et al. 2002, Sanchez-Alonso et al. 2002, Figueiredo \& Custódio-Filho 2003, Beisiegel 2006, 2007, Rocca et al. 2006, Antunes et al. 2006, 2007, 2011, Francisco et al. 2008, Rocca \& Sazima 2008, Silva et al. 2010, Bernardo et al. 2011, Oliveira et al. 2011).

\section{Coleta e análise de dados}

As aves foram amostradas pelo método de trajetos de distância ilimitada (Bibby et al. 1992), que consistiu em deslocar-se lentamente, a aproximadamente $1 \mathrm{~km} / \mathrm{h}$, pelas trilhas e estradas disponíveis, registrando-se cada indivíduo contatado. Foram utilizados binóculos $8 \times 40$ para auxiliar na identificação das espécies e gravador profissional PMD222 Marantz com microfone Sennheiser ME66/ K6C para registrar vocalizações e outras manifestações sonoras, a fim de documentar a presença das espécies. As coordenadas das áreas amostradas foram obtidas com GPS Garmin e-Trex Summit. A nomenclatura científica adotada foi a do Comitê Brasileiro de Registros Ornitológicos (Comitê... 2011). As espécies consideradas ameaçadas de extinção são aquelas listadas em São Paulo (2008b).

O esforço amostral totalizou 175 dias em campo, entre abril de 2006 e dezembro de 2009, 1002 horas e, aproximadamente, $800 \mathrm{~km}$ percorridos a pé. Em relação às fitofisionomias o esforço amostral foi assim distribuído: $400 \mathrm{~h}$ para a Floresta Montana, $380 \mathrm{~h}$ para a Floresta Submontana, $100 \mathrm{~h}$ para as capoeiras, $60 \mathrm{~h}$ para o reflorestamento, $42 \mathrm{~h}$ para os gramados e jardins, e $20 \mathrm{~h}$ para os açudes e brejos.

A riqueza local foi estimada pelo método Bootstrap por meio do Programa EstimateS (Colwell 2009) e comparada ao valor obtido. Tal método foi selecionado por considerar os dados de abundância relativa de todas as espécies. Nessa análise, foi considerada como unidade amostral a campanha mensal, incluindo-se separadamente amostras de áreas montanas e submontanas obtidas em um mesmo mês e totalizando-se 50 amostras. Variações na riqueza e total de contatos ao longo do ano foram avaliadas agrupando-se os meses em trimestres, considerando-se as variações de temperatura e precipitação do clima local (São Paulo 2008a): dezembro-fevereiro, março-maio, 


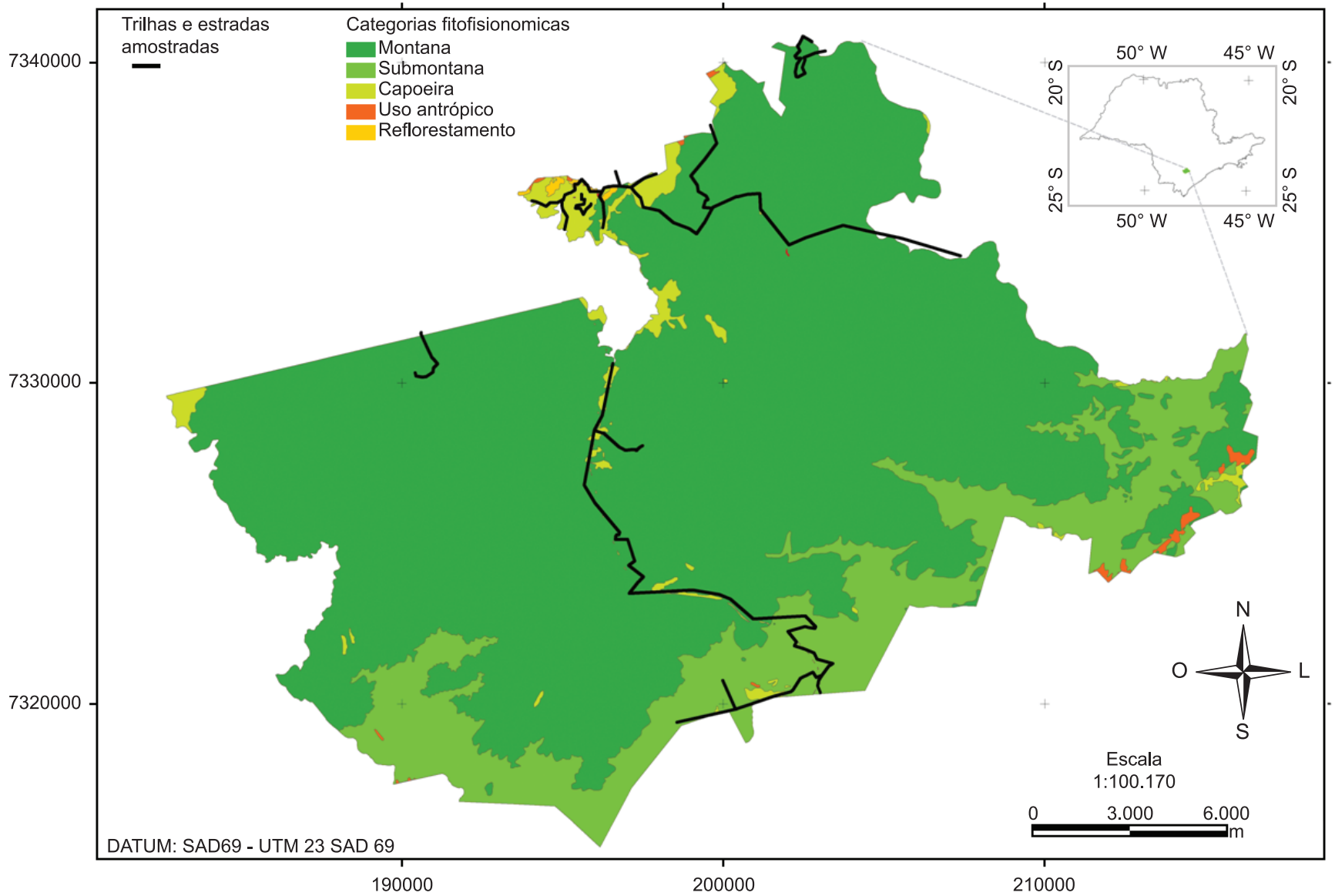

Figura 1. Principais fitofisionomias presentes no Parque Estadual Carlos Botelho e localização das trilhas e estradas amostradas.

Figure 1. Main vegetation types in Carlos Botelho State Park and location of sampled trails and roads.

junho-agosto e setembro-novembro. Valores médios de contatos são apresentados com os desvios padrões e foram comparados através do teste Mann-Whitney (U) ou de ANOVA de um fator (F). Para o último tipo de teste os dados sofreram transformação logarítmica. A correlação entre valores médios mensais de riqueza e total de contatos foi avaliada por meio do teste de correlação de postos de Spearman (rs). Os testes estatísticos foram efetuados com o programa BioEstat 5.0 (Ayres et al. 2008) e os resultados foram considerados significativos para $\mathrm{p}<0,01$.

\section{Resultados}

Foram obtidos 30353 contatos para 331 espécies (Tabela 1). Duzentas e uma foram gravadas, resultando na documentação de $60 \%$ da assembleia amostrada. A distribuição das espécies em classes de abundância relativa aponta que a maioria das espécies foi pouco contatada (Figura 2). A estimativa de riqueza indica um acréscimo esperado de $5 \%$ com a continuação da amostragem (Figura 3 ).

Noventa espécies $(27 \%)$ foram encontradas apenas nas fitofisionomias antropogênicas (Figura 4) e foram significativamente menos contatadas do que as demais espécies (média $=22,18 \pm 62,02$ contatos), $\mathrm{U}=4474,50 \mathrm{p}<0,00001$, representando $6 \%(\mathrm{~N}=1996)$ do total de contatos obtidos.

O período compreendido entre setembro e novembro apresentou os maiores valores de riqueza (Tabela 2), sendo que no total 278 espécies (84\%) foram detectadas nesse trimestre (Tabela 1). A riqueza média desse trimestre diferiu significativamente em relação ao trimestre junho-agosto $(\mathrm{F}=5,45 \mathrm{p}=0,0034$; teste Tukey $\mathrm{Q}=5,56 \mathrm{p}$
$<0,01)$, mas não dos demais. A comparação entre médias de contatos trimestrais não foi possível, pois as variâncias foram desiguais. Não houve correlação entre as médias mensais de riqueza e total de contatos, $r s=0,02 p=0,95$. A distribuição dos contatos ao longo do ano indica que pelo menos 17 espécies (5\%) podem ser consideradas localmente migratórias, sendo que Pyrocephalus rubinus (Boddaert, 1783) foi detectado entre maio e outubro e as demais entre agosto e abril (Tabela 1).

Vinte e cinco espécies (7\%) são consideradas ameaçadas de extinção (18 vulneráveis, quatro em perigo e três criticamente em perigo). Estas espécies (média $=39,48 \pm 51,70$ contatos) não diferiram significativamente no total de contatos obtidos em relação às espécies de menor preocupação conservacionista $(\mathrm{N}=306$ média $=95,96 \pm 181,34$ contatos), $U=3362,5 p=0,31$. Também, não diferiram significativamente no número de fitofisionomias ocupadas, $\mathrm{U}=3121,50 \mathrm{p}=0,12$ (Figura 5).

\section{Discussão}

Assim como para outras áreas de alta diversidade, foram necessários vários anos de amostragem para se alcançar a caracterização da avifauna apresentada (Cohn-Haft et al. 1997). A avifauna conhecida no interior dessa unidade de conservação totaliza 370 espécies, quando são considerados os registros obtidos e publicados por outros pesquisadores (Antunes et al. 2006). Esta riqueza registrada corresponde a $47 \%$ da avifauna conhecida para o estado de São Paulo (Silveira \& Uezu 2011). Ressalte-se que a maior parte da área do PECB ainda não foi amostrada devido a questões 
Tabela 1. Espécies de aves registradas no Parque Estadual Carlos Botelho entre abril de 2006 e dezembro de 2009, com o total de contatos obtidos, o status de conservação, as fitofisionomias e meses de ocorrência. ${ }^{*}=$ espécie gravada $;{ }^{\mathrm{m}}=$ espécie migratória; $\mathrm{CR}=$ Criticamente em Perigo; $\mathrm{EN}=\mathrm{Em}$ Perigo; $\mathrm{NT}=$ quase Ameaçada; $\mathrm{VU}=$ Vulnerável; $\mathrm{AB}=$ açudes e brejos; $\mathrm{CP}=$ capoeiras; $\mathrm{GJ}=$ gramados e jardins; $\mathrm{FM}=$ Floresta Ombrófila Densa Montana; FS = Floresta Ombrófila Densa Submontana; $\mathrm{RA}=$ reflorestamento com pinheiros-do-Paraná; $\mathrm{MR}=$ margens de riachos; $\mathrm{SO}=$ sobrevoando e $\mathrm{TA}=$ taquarais.

Table 1. Bird species recorded in Carlos Botelho State Park between April 2006 and December 2009, with total contacts made, conservation status, vegetation types and months of occurrence. ${ }^{*}=$ audio-recorded; ${ }^{\mathrm{m}}=$ migratory; $\mathrm{CR}=$ critically endangered; $\mathrm{EN}=$ endangered; $\mathrm{NT}=$ near threatened; $\mathrm{VU}=$ vulnerable; $\mathrm{AB}=$ ponds and swamps; $\mathrm{CP}=$ secondary forest; $\mathrm{GJ}=$ lawns and gardens; $\mathrm{FM}=$ lower highlands evergreen tropical forest; $\mathrm{FS}=$ lower plains evergreen tropical forest and $\mathrm{RA}=$ Parana pine reforestation. $\mathrm{MR}=$ river edges; $\mathrm{SO}=$ flying over and $\mathrm{TA}=$ bamboo clumps.

\begin{tabular}{|c|c|c|c|c|c|}
\hline Espécie & Nome popular & Contatos & Status & Fitofisionomias & Meses \\
\hline \multicolumn{6}{|l|}{ Tinamiformes } \\
\hline \multicolumn{6}{|l|}{ Tinamidae } \\
\hline Tinamus solitarius (Vieillot, 1819)* & macuco & 187 & VU & FM FS CP RA & Jan-Dez \\
\hline Crypturellus obsoletus (Temminck, 1815)* & inhambu-guaçu & 90 & & FM FS CP RA & Jan-Dez \\
\hline Crypturellus noctivagus (Wied, 1820)* & jaó-do-sul & 76 & EN & FM FS CP & Mar, Maio, Jul-Dez \\
\hline Crypturellus tataupa (Temminck, 1815) & inhambu-chintã & 1 & & FS & Ago \\
\hline \multicolumn{6}{|l|}{ Anseriformes } \\
\hline \multicolumn{6}{|l|}{ Anatidae } \\
\hline Dendrocygna viduata (Linnaeus, 1766) & irerê & 2 & & $\mathrm{AB}$ & Nov e Out \\
\hline Cairina moschata (Linnaeus, 1758) & pato-do-mato & 1 & & $\mathrm{AB}$ & Ago \\
\hline \multicolumn{6}{|l|}{ Galliformes } \\
\hline \multicolumn{6}{|l|}{ Cracidae } \\
\hline Penelope obscura Temminck, 1815* & jacuguaçu & 74 & & FM FS CP RA & Jan-Dez \\
\hline Aburria jacutinga (Spix, 1825)* & jacutinga & 29 & $\mathrm{CR}$ & FM FS CP & Jan, Fev, Abr-Out e Dez \\
\hline \multicolumn{6}{|l|}{ Odontophoridae } \\
\hline Odontophorus capueira (Spix, 1825)* & uru & 136 & NT & FM FS CP RA & Jan-Dez \\
\hline \multicolumn{6}{|l|}{ Suliformes } \\
\hline \multicolumn{6}{|l|}{ Phalacrocoracidae } \\
\hline Phalacrocorax brasilianus (Gmelin, 1789) & biguá & 2 & & $\mathrm{AB}$ & Abr e Out \\
\hline \multicolumn{6}{|l|}{ Pelecaniformes } \\
\hline \multicolumn{6}{|l|}{ Ardeidae } \\
\hline Tigrisoma lineatum (Boddaert, 1783) & socó-boi & 1 & & FS (MR) & Out \\
\hline Tigrisoma fasciatum (Such, 1825) & socó-boi-escuro & 1 & $\mathrm{CR}$ & FM (MR) & Jun \\
\hline Bubulcus ibis (Linnaeus, 1758) & garça-vaqueira & 25 & & GJ & Maio, Jun, Ago \\
\hline Ardea cocoi Linnaeus, 1766 & garça-moura & 1 & & $\mathrm{AB}$ & \\
\hline Ardea alba Linnaeus, 1758 & garça-branca-grande & 2 & & $\mathrm{AB}$ & Ago e Out \\
\hline Syrigma sibilatrix (Temminck, 1824)* & maria-faceira & 2 & & GJ & Jan, Abr-Jun-Dez \\
\hline \multicolumn{6}{|l|}{ Threskiornithidae } \\
\hline Theristicus caudatus (Boddaert, 1783) & curicaca & 3 & & GJ RA & Set \\
\hline \multicolumn{6}{|l|}{ Cathartiformes } \\
\hline \multicolumn{6}{|l|}{ Cathartidae } \\
\hline Cathartes aura (Linnaeus, 1758) & $\begin{array}{l}\text { urubu-de-cabeça- } \\
\text { vermelha }\end{array}$ & 18 & & FM FS CP (SO) & Fev, Abr, Jun-Nov \\
\hline Coragyps atratus (Bechstein, 1793) & urubu-preto & 231 & & FM FS CP (SO) & Jan-Dez \\
\hline \multicolumn{6}{|l|}{ Accipitriformes } \\
\hline \multicolumn{6}{|l|}{ Accipitridae } \\
\hline Leptodon cayanensis (Latham, 1790) & $\begin{array}{l}\text { gavião-de-cabeça- } \\
\text { cinza }\end{array}$ & 1 & & FM & Out \\
\hline Elanoides forficatus (Linnaeus, 1758) & gavião-tesoura & 4 & & FM & Jan-Fev \\
\hline Elanus leucurus (Vieillot, 1818) & gavião-peneira & 1 & & GJ & Nov \\
\hline Harpagus diodon $(\text { Temminck, } 1823)^{\mathrm{m}}$ & gavião-bombachinha & 14 & & FM FS & Ago-Fev \\
\hline Accipiter poliogaster (Temminck, 1824) & tauató-pintado & 2 & NT & FM & Jan \\
\hline Accipiter superciliosus (Linnaeus, 1766) & gavião-miudinho & 1 & NT & FM & Abr \\
\hline Geranospiza caerulescens (Vieillot, 1817) & gavião-pernilongo & 2 & & FM RA & Ago e Out \\
\hline Heterospizias meridionalis (Latham, 1790) & gavião-caboclo & 1 & & RA & Jan \\
\hline Amadonastur lacernulatus (Temminck, 1827) & gavião-pombo-pequeno & 1 & VU & FM & Out \\
\hline Rupornis magnirostris (Gmelin, 1788)* & gavião-carijó & 78 & & CP RA GJ & Jan-Dez \\
\hline Parabuteo leucorrhous (Quoy \& Gaimard, 1824) & gavião-de-sobre-branco & 9 & & FM & Dez-Mar \\
\hline
\end{tabular}


Tabela 1. Continuação...

\begin{tabular}{llccll}
\hline \multicolumn{1}{c}{ Espécie } & \multicolumn{1}{c}{ Nome popular } & Contatos & Status & Fitofisionomias & Meses \\
\hline Geranoaetus albicaudatus Vieillot, 1816 & gavião-de-rabo-branco & 1 & & GJ & Jun \\
Pseudastur polionotus (Kaup, 1847) & gavião-pombo-grande & 31 & VU & FM & Jan-Abr, Jun-Dez \\
Buteo brachyurus Vieillot, 1816 & gavião-de-rabo-curto & 4 & & FM CP (SO) & Mar, Ago e Set \\
Spizaetus tyrannus (Wied, 1820)* & gavião-pega-macaco & 13 & VU & FM FS & Jan-Jun, Set e Nov \\
Spizaetus melanoleucus (Vieillot, 1816) & gavião-pato & 1 & EN & FM & Set \\
Spizaetus ornatus (Daudin, 1800) & gavião-de-penacho & 1 & CR & FS & Maio
\end{tabular}

Falconiformes

Falconidae

Caracara plancus (Miller, 1777)

Milvago chimachima (Vieillot, 1816)*

Herpetotheres cachinnans (Linnaeus, 1758)

Micrastur ruficollis (Vieillot, 1817)*

Micrastur semitorquatus (Vieillot, 1817)*

Falco sparverius Linnaeus, 1758

Falco femoralis Temminck, 1822

Gruiformes

Aramidae

Aramus guarauna (Linnaeus, 1766)

Rallidae

Aramides saracura (Spix, 1825)*

Amaurolimnas concolor (Gosse, 1847)

Laterallus melanophaius (Vieillot, 1819)

Pardirallus nigricans (Vieillot, 1819)

Cariamiformes

Cariamidae

Cariama cristata (Linnaeus, 1766)

Charadriiformes

Charadriidae

Vanellus chilensis (Molina, 1782)*

Columbiformes

Columbidae

Columbina talpacoti (Temminck, 1811)*

Patagioenas picazuro (Temminck, 1813)*

Patagioenas cayennensis (Bonnaterre, 1792)

Patagioenas plumbea (Vieillot, 1818)*

Zenaida auriculata (Des Murs, 1847)*

Leptotila verreauxi Bonaparte, 1855*

Leptotila rufaxilla (Richard \& Bernard, 1792)

Geotrygon montana (Linnaeus, 1758)*

Psittaciformes

Psittacidae

Pyrrhura frontalis (Vieillot, 1817)*

Forpus xanthopterygius (Spix, 1824)*

Brotogeris tirica (Gmelin, 1788)*

Touit melanonotus (Wied, 1820)

Pionopsitta pileata (Scopoli, 1769)*

Pionus maximiliani (Kuhl, 1820)*

Triclaria malachitacea (Spix, 1824)*

Cuculiformes

Cuculidae

Piaya cayana (Linnaeus, 1766)

Crotophaga ani Linnaeus, 1758

Guira guira (Gmelin, 1788)*

caracará

carrapateiro

acauã

falcão-caburé

falcão-relógio

quiriquiri

falcão-de-coleira

carão

saracura-do-mato

116

saracura-lisa

sanã-parda

saracura-preta

seriema

3

quero-quero

10

rolinha-roxa

4

asa-branca

210

pomba-galega

20

pomba-amargosa

141

avoante

juriti-pupu

juriti-gemedeira

juriti-piranga

tiriba-de-testa-vermelha 1406

tuim 237

periquito-rico $\quad 843$

apuim-de-costas-pretas $\quad 17$

cuiú-cuiú 194

maitaca-verde $\quad 774$

sabiá-cica

alma-de-gato

anu-preto

anu-branco

$\begin{array}{ll}\text { CP RA GJ } & \text { Jan, Abr-Jun, Agc } \\ \text { CP RA GJ } & \text { Jan-Dez } \\ \text { CP RA } & \text { Jan, Fev, Abr, Set } \\ \text { FM FS CP RA } & \text { Jan-Dez } \\ \text { FM FS RA } & \text { Jan-Out, Dez } \\ \text { GJ } & \text { Fev, Jun e Set } \\ \text { GJ } & \text { Abr }\end{array}$

FS (MR) Jul

FM FS CP RA Jan-Jun, Ago-Dez

FS (MR) Out

$\mathrm{AB} \quad \mathrm{Dez}$

$\mathrm{AB} \quad \mathrm{Mar}$

GJ Jan-Dez

GJ Jan-Dez

GJ

CP GJ RA Jan-Dez

FS CP Set-Dez

FM FS RA Jan-Dez

GJ Jan-Jun, Ago-Dez

CP Jan, Abr, Jul-Dez

CP RA Out-Nov

FM FS Fev, Abr, Jun, Ago-Dez

FM FS CP GJ RA Jan-Dez

FM FS CP GJ Jan-Dez

FM FS CP GJ RA Jan-Dez

VU FS Fev

FM FS Jan-Dez

FM FS CP GJ RA Jan-Dez

VU FM FS CP Jan-Set, Nov e Dez

$\begin{array}{ll}\text { FM FS CP GJ RA } & \text { Jan-Dez } \\ \text { GJ } & \text { Jan, Jun e Ago } \\ \text { GJ } & \text { Jan, Fev, Abr-Jun, Ago- } \\ & \text { Dez }\end{array}$


Tabela 1. Continuação...

\begin{tabular}{|c|c|c|c|c|c|}
\hline Espécie & Nome popular & Contatos & Status & Fitofisionomias & Meses \\
\hline Tapera naevia (Linnaeus, 1766) & saci-do-campo & 1 & & $\mathrm{CP}$ & Jul \\
\hline \multicolumn{6}{|l|}{ Strigiformes } \\
\hline \multicolumn{6}{|l|}{ Tytonidae } \\
\hline Tyto alba (Scopoli, 1769) & suindara & 2 & & GJ & Maio e Ago \\
\hline \multicolumn{6}{|l|}{ Strigidae } \\
\hline Megascops choliba (Vieillot, 1817) & corujinha-do-mato & 4 & & GJ & Jan, Abr, Maio e nov \\
\hline $\begin{array}{l}\text { Pulsatrix koeniswaldiana } \\
\text { (Bertoni \& Bertoni, 1901) }\end{array}$ & $\begin{array}{l}\text { murucututu-de- } \\
\text { barriga-amarela }\end{array}$ & 7 & & FM RA & Jan, Mar, Abr, Ago-Out \\
\hline Strix hylophila Temminck, 1825* & coruja-listrada & 7 & & FM RA & Mar, Abr e Out \\
\hline Strix virgata (Cassin, 1849) & coruja-de-bigodes & 1 & & FM & Mar \\
\hline Glaucidium minutissimum (Wied, 1830)* & caburé-miudinho & 13 & & FM FS & Mar, Abr, Jul-Set, Dez \\
\hline Athene cunicularia (Molina, 1782) & coruja-buraqueira & 2 & & GJ & Abr e Set \\
\hline \multicolumn{6}{|l|}{ Caprimulgiformes } \\
\hline \multicolumn{6}{|l|}{ Nyctibiidae } \\
\hline Nyctibius griseus (Gmelin, 1789)* & urutau & 12 & & FM FS RA & Fev, Abr, Ago, Out-Dez \\
\hline \multicolumn{6}{|l|}{ Caprimulgidae } \\
\hline Nyctiphrynus ocellatus (Tschudi, 1844) & bacurau-ocelado & 2 & & FS & Maio \\
\hline Lurocalis semitorquatus (Gmelin, 1789)* m & tuju & 30 & & $\begin{array}{l}\text { FM FS CP GJ RA } \\
(\mathrm{SO})\end{array}$ & Set-Abr \\
\hline Hydropsalis albicollis (Gmelin, 1789)* & curiango & 37 & & CP GJ & Jan, Mar-Dez \\
\hline Hydropsalis torquata (Gmelin, 1789) & bacurau-tesoura & 6 & & CP GJ RA & Abr, Jul e Ago \\
\hline Hydropsalis forcipata (Nitzsch, 1840) & bacurau-tesourão & 1 & NT & $\mathrm{CP}$ & Nov \\
\hline \multicolumn{6}{|l|}{ Apodiformes } \\
\hline \multicolumn{6}{|l|}{ Apodidae } \\
\hline Cypseloides fumigatus (Streubel, 1848) & taperuçu-preto & 50 & & FS & Jun \\
\hline Streptoprocne zonaris (Shaw, 1796) & taperuçu-de-coleira & 110 & & $\begin{array}{l}\text { FM FS CP GJ RA } \\
(\mathrm{SO})\end{array}$ & Jan-Dez \\
\hline Chaetura cinereiventris Sclater, 1862* & taperá-de-barriga-cinza & 476 & & $\begin{array}{l}\text { FM FS CP GJ RA } \\
(\mathrm{SO})\end{array}$ & Jan-Dez \\
\hline Chaetura meridionalis Hellmayr, 1907* & taperá-do-temporal & 22 & & FS CP (SO) & Set-Nov \\
\hline Panyptila cayennensis (Gmelin, 1789) & taperá-estofador & 3 & & FS & Maio e Set \\
\hline \multicolumn{6}{|l|}{ Trochilidae } \\
\hline Ramphodon naevius (Dumont, 1818)* & beija-flor-rajado & 425 & & FS CP & Jan-Dez \\
\hline Phaethornis squalidus (Temminck, 1822)* & rabo-branco-miúdo & 27 & & FM FS & Jan-Ago, Out e Dez \\
\hline Phaethornis pretrei (Lesson \& Delattre, 1839) & rabo-branco-acanelado & 1 & & GJ & Maio \\
\hline Phaethornis eurynome (Lesson, 1832)* & $\begin{array}{l}\text { rabo-branco-de- } \\
\text { garganta-rajada }\end{array}$ & 130 & & FM FS CP GJ RA & Jan-Dez \\
\hline Eupetomena macroura (Gmelin, 1788) & beija-flor-tesoura & 14 & & GJ & $\begin{array}{l}\text { Jan-Fev, Maio-Jun, } \\
\text { Ago-Set, Nov-Dez }\end{array}$ \\
\hline Aphantochroa cirrochloris (Vieillot, 1818)* & beija-flor-cinza & 4 & & FS CP & Jan, Fev, Jun e Dez \\
\hline Florisuga fusca (Vieillot, 1817) & beija-flor-preto & 78 & & FM FS CP GJ RA & Jul, Set-Abr \\
\hline Colibri serrirostris (Vieillot, 1816) & $\begin{array}{l}\text { beija-flor-de-orelha- } \\
\text { violeta }\end{array}$ & 1 & & GJ & Dez \\
\hline Anthracothorax nigricollis (Vieillot, 1817) ${ }^{\mathrm{m}}$ & beija-flor-de-veste-preta & 10 & & FS GJ & Out-Abr \\
\hline Lophornis chalybeus (Vieillot, 1823)* & topetinho-verde & 3 & & FS GJ & Jan, Abr e Jun \\
\hline Chlorostilbon lucidus (Shaw, 1812) & $\begin{array}{l}\text { esmeralda-de-bico- } \\
\text { vermelho }\end{array}$ & 17 & & GJ & Jan, Fev, Set-Dez \\
\hline Thalurania glaucopis (Gmelin, 1788)* & $\begin{array}{l}\text { beija-flor-de-fronte- } \\
\text { violeta }\end{array}$ & 240 & & FM FS CP GJ RA & Jan-Dez \\
\hline Leucochloris albicollis (Vieillot, 1818) & $\begin{array}{l}\text { beija-flor-de-papo- } \\
\text { branco }\end{array}$ & 13 & & FM FS CP GJ & Fev-Maio, Set, Nov-Dez \\
\hline Amazilia versicolor (Vieillot, 1818) & $\begin{array}{l}\text { beija-flor-de-banda- } \\
\text { branca }\end{array}$ & 15 & & FM FS CP GJ & Jan, Fev, Set-Dez \\
\hline Amazilia fimbriata (Gmelin, 1788) & $\begin{array}{l}\text { beija-flor-de-garganta- } \\
\text { verde }\end{array}$ & 6 & & FM FS & Jan, Out e Dez \\
\hline
\end{tabular}


Tabela 1. Continuação...

\begin{tabular}{|c|c|c|c|c|c|}
\hline Espécie & Nome popular & Contatos & Status & Fitofisionomias & Meses \\
\hline Amazilia lactea (Lesson, 1832) & beija-flor-de-peito-azul & 4 & & GJ & Jan, Maio e Dez \\
\hline Clytolaema rubricauda (Boddaert, 1783)* & beija-flor-rubi & 8 & & FM CP & $\begin{array}{l}\text { Fev, Abr, Jun, Ago, Set } \\
\text { e Dez }\end{array}$ \\
\hline Heliomaster squamosus (Temminck, 1823) & $\begin{array}{l}\text { bico-reto-de-banda- } \\
\text { branca }\end{array}$ & 1 & & GJ & Dez \\
\hline \multicolumn{6}{|l|}{ Trogoniformes } \\
\hline \multicolumn{6}{|l|}{ Trogonidae } \\
\hline Trogon viridis Linnaeus, $1766^{*}$ & $\begin{array}{l}\text { surucuá-de-barriga- } \\
\text { dourada }\end{array}$ & 182 & & FM FS CP & Jan-Dez \\
\hline Trogon surrucura Vieillot, 1817* & surucuá-variado & 152 & & FM CP RA & Jan-Dez \\
\hline Trogon rufus Gmelin, 1788* & $\begin{array}{l}\text { surucuá-de-barriga- } \\
\text { amarela }\end{array}$ & 166 & & FM FS CP RA & Jan-Dez \\
\hline \multicolumn{6}{|l|}{ Coraciiformes } \\
\hline \multicolumn{6}{|l|}{ Alcedinidae } \\
\hline Megaceryle torquata (Linnaeus, 1766) & $\begin{array}{l}\text { martim-pescador- } \\
\text { grande }\end{array}$ & 8 & & AB GJ (SO) & Abr, Jun-Set \\
\hline Chloroceryle amazona (Latham, 1790) & martim-pescador-verde & 7 & & AB FS (MR) & Fev-Abr, Jul, Ago e Out \\
\hline Chloroceryle americana (Gmelin, 1788) & $\begin{array}{l}\text { martim-pescador- } \\
\text { pequeno }\end{array}$ & 11 & & AB FS (MR) & $\begin{array}{l}\text { Fev, Mar, Maio, Set, } \\
\text { Out e Dez }\end{array}$ \\
\hline Chloroceryle inda (Linnaeus, 1766) & $\begin{array}{l}\text { martim-pescador-da- } \\
\text { mata }\end{array}$ & 4 & NT & FM FS (MR) & Jan, Mar e Set \\
\hline \multicolumn{6}{|l|}{ Momotidae } \\
\hline Baryphthengus ruficapillus (Vieillot, 1818)* & juruva-verde & 125 & & FM FS CP & Jan-Dez \\
\hline \multicolumn{6}{|l|}{ Galbuliformes } \\
\hline \multicolumn{6}{|l|}{ Bucconidae } \\
\hline Notharchus swainsoni (Gray, 1846) & $\begin{array}{l}\text { macuru-de-barriga- } \\
\text { castanha }\end{array}$ & 3 & NT & FS & Fev e Abr \\
\hline Malacoptila striata (Spix, 1824) & barbudo-rajado & 11 & & FM FS CP & $\begin{array}{l}\text { Fev, Mar, Jun, jul, Set, } \\
\text { Nov e Dez }\end{array}$ \\
\hline \multicolumn{6}{|l|}{ Piciformes } \\
\hline \multicolumn{6}{|l|}{ Ramphastidae } \\
\hline Ramphastos vitellinus Lichtenstein, 1823* & tucano-de-bico-preto & 97 & & FM FS CP & Jan-Dez \\
\hline Ramphastos dicolorus Linnaeus, 1766* & tucano-de-bico-verde & 293 & & FM FS CP GJ RA & Jan-Dez \\
\hline Selenidera maculirostris (Lichtenstein, 1823)* & araçari-poca & 55 & VU & FM FS CP & Jan-Dez \\
\hline Pteroglossus bailloni (Vieillot, 1819) & araçari-banana & 22 & VU & FM FS & $\begin{array}{l}\text { Fev, Abr-Jun, Set, Nov } \\
\text { e Dez }\end{array}$ \\
\hline \multicolumn{6}{|l|}{ Picidae } \\
\hline Picumnus temminckii Lafresnaye, 1845* & $\begin{array}{l}\text { pica-pau-anão-de- } \\
\text { coleira }\end{array}$ & 198 & & FM FS CP GJ RA & Jan-Dez \\
\hline Melanerpes candidus (Otto, 1796)* & pica-pau-branco & 3 & & GJ & Maio e Jun \\
\hline Melanerpes flavifrons (Vieillot, 1818)* & $\begin{array}{l}\text { benedito-de-testa- } \\
\text { amarela }\end{array}$ & 135 & & FM FS CP GJ & Jan-Dez \\
\hline Veniliornis spilogaster (Wagler, 1827)* & pica-pau-manchado & 159 & & FM FS CP GJ RA & Jan-Dez \\
\hline Piculus flavigula (Boddaert, 1783) & pica-pau-bufador & 17 & & FS & Mar-Jul e Set \\
\hline Piculus aurulentus (Temminck, 1821)* & pica-pau-dourado & 18 & & FM RA & Fev, Abr, Jun-Nov \\
\hline Colaptes melanochloros (Gmelin, 1788)* & pica-pau-carijó & 11 & & FM FS CP GJ & Mar, Ago, Set e Dez \\
\hline Colaptes campestris (Vieillot, 1818) & pica-pau-do-campo & 3 & & GJ & Fev, Abr-Jun, Ago-Nov \\
\hline Celeus flavescens (Gmelin, 1788)* & pica-pau-velho & 215 & & FM FS CP GJ RA & Jan-Dez \\
\hline Dryocopus lineatus (Linnaeus, 1766)* & $\begin{array}{l}\text { pica-pau-de-banda- } \\
\text { branca }\end{array}$ & 24 & & CP GJ RA & Jan-Mar, Jun, Ago-Dez \\
\hline Campephilus robustus (Lichtenstein, 1818)* & pica-pau-rei & 54 & NT & FM FS CP RA & Jan-Set, Nov-Dez \\
\hline \multicolumn{6}{|l|}{ Passeriformes } \\
\hline \multicolumn{6}{|l|}{ Thamnophilidae } \\
\hline Terenura maculata (Wied, 1831)* & zidedê & 293 & & FM FS RA & Jan-Dez \\
\hline Myrmeciza squamosa Pelzeln, 1868* & formigueiro-da-grota & 253 & & FM FS CP RA & Jan-Dez \\
\hline
\end{tabular}


Tabela 1. Continuação...

\begin{tabular}{|c|c|c|c|c|c|}
\hline Espécie & Nome popular & Contatos & Status & Fitofisionomias & Meses \\
\hline Myrmotherula gularis (Spix, 1825)* & $\begin{array}{l}\text { choquinha-de- } \\
\text { garganta-pintada }\end{array}$ & 458 & & FM FS RA & Jan-Dez \\
\hline Myrmotherula minor Salvadori, 1864 & choquinha-pequena & 2 & VU & FS & Jun e Set \\
\hline Myrmotherula unicolor (Menetries, 1835) & choquinha-cinzenta & 25 & VU & FM FS & Fev, Mar, Maio-Dez \\
\hline Dysithamnus stictothorax (Temminck, 1823)* & $\begin{array}{l}\text { choquinha-de-peito- } \\
\text { pintado }\end{array}$ & 87 & NT & FM FS CP & Jan-Dez \\
\hline Dysithamnus mentalis (Temminck, 1823)* & choquinha-lisa & 340 & & FM FS CP RA & Jan-Dez \\
\hline Dysithamnus xanthopterus Burmeister, 1856* & $\begin{array}{l}\text { choquinha-de-asa- } \\
\text { ferrugem }\end{array}$ & 3 & & FM CP & Out e Dez \\
\hline $\begin{array}{l}\text { Herpsilochmus rufimarginatus } \\
\text { (Temminck, 1822)* }\end{array}$ & $\begin{array}{l}\text { chorozinho-de-asa- } \\
\text { vermelha }\end{array}$ & 28 & & FM FS & Jan, Mar-Nov \\
\hline Thamnophilus caerulescens Vieillot, 1816* & choca-da-mata & 70 & & FM FS GJ CP & Jan, Fev, Abr-Dez \\
\hline Hypoedaleus guttatus (Vieillot, 1816)* & chocão-carijó & 169 & & FM FS CP RA & Jan-Dez \\
\hline Batara cinerea (Vieillot, 1819)* & matracão & 54 & & $\begin{array}{l}\text { FM FS CP RA } \\
\text { (TA) }\end{array}$ & Jan-Nov \\
\hline Mackenziaena leachii (Such, 1825) & borralhara-assobiadora & 7 & & FM FS (TA) & Mar, Maio e Dez \\
\hline Mackenziaena severa (Lichtenstein, 1823)* & borralhara-preta & 30 & & FM FS CP (TA) & Fev, Abr-Dez \\
\hline Pyriglena leucoptera (Vieillot, 1818)* & olho-de-fogo-do-sul & 346 & & FM FS CP RA & Jan-Dez \\
\hline Drymophila ferruginea (Temminck, 1822)* & dituí & 63 & & FM FS CP (TA) & Jan-Dez \\
\hline Drymophila rubricollis (Bertoni, 1901) & trovoada-de-bertoni & 3 & & FM FS (TA) & Jul, Set e Dez \\
\hline Drymophila ochropyga (Hellmayr, 1906)* & $\begin{array}{l}\text { trovoada-de-dorso- } \\
\text { vermelho }\end{array}$ & 24 & & FM FS (TA) & Fev-Jul, Set-Dez \\
\hline Drymophila malura (Temminck, 1825)* & trovoada-carijó & 14 & & FM FS CP (TA) & Jan-Abr, Ago-Dez \\
\hline Drymophila squamata (Lichtenstein, 1823)* & pintadinho & 19 & & FS & $\begin{array}{l}\text { Mar, Maio, Jul, Ago } \\
\text { e Nov }\end{array}$ \\
\hline \multicolumn{6}{|l|}{ Conopophagidae } \\
\hline Conopophaga lineata (Wied, 1831)* & chupa-dente-marrom & 76 & & FM FS CP RA & Jan-Dez \\
\hline Conopophaga melanops (Vieillot, 1818)* & chupa-dente-mascarado & 35 & & FM FS & Fev, Abr-Set, Nov e Dez \\
\hline \multicolumn{6}{|l|}{ Grallariidae } \\
\hline Grallaria varia (Boddaert, 1783)* & tovacuçu & 79 & & FM FS RA & Jan-Dez \\
\hline \multicolumn{6}{|l|}{ Rhinocryptidae } \\
\hline Merulaxis aff. ater Lesson, 1830* & bigodudo-preto & 43 & NT & FS CP & Jan-Dez \\
\hline Eleoscytalopus indigoticus (Wied, 1831)* & macuquinho-perereca & 141 & & $\begin{array}{l}\text { FM FS CP RA } \\
\text { (TA) }\end{array}$ & Jan-Dez \\
\hline Scytalopus aff. speluncae (Ménétriès, 1835)* & macuquinho-preto & 9 & & FM FS (TA) & Mar, Abr, Ago-Nov \\
\hline Psilorhamphus guttatus (Ménétriès, 1835)* & macuquinho-pintado & 44 & & FM FS CP (TA) & Fev-Dez \\
\hline \multicolumn{6}{|l|}{ Formicariidae } \\
\hline Formicarius colma Boddaert, 1783* & galinha-do-mato & 90 & & FS CP & Fev-Dez \\
\hline Chamaeza campanisona (Lichtenstein, 1823)* & tovaca-campainha & 246 & & FM FS RA & Jan-Dez \\
\hline Chamaeza meruloides Vigors, 1825 & tovaca-cantadora & 16 & & FM FS & $\begin{array}{l}\text { Fev, Abr-Jul, Set, Out } \\
\text { e Dez }\end{array}$ \\
\hline \multicolumn{6}{|l|}{ Scleruridae } \\
\hline Sclerurus mexicanus Sclater, 1857* & $\begin{array}{l}\text { vira-folha-de-peito- } \\
\text { vermelho }\end{array}$ & 6 & VU & FS & Mar, Maio, Jul e Ago \\
\hline Sclerurus scansor (Menetries, 1835)* & vira-folha-vermelho & 32 & & FM FS CP RA & Jan-Jun, Set-Nov \\
\hline \multicolumn{6}{|l|}{ Dendrocolaptidae } \\
\hline Dendrocincla turdina (Lichtenstein, 1820)* & arapaçu-liso & 204 & & FM FS & Jan-Dez \\
\hline Sittasomus griseicapillus (Vieillot, 1818)* & arapaçu-verde & 321 & & FM FS CP GJ RA & Jan-Dez \\
\hline Xiphorhynchus fuscus (Vieillot, 1818)* & arapaçu-rajado & 271 & & FM FS CP RA & Jan-Dez \\
\hline Campylorhamphus falcularius (Vieillot, 1822) & arapaçu-alfange & 23 & & FM FS (TA) & Maio-Dez \\
\hline $\begin{array}{l}\text { Lepidocolaptes falcinellus } \\
\text { (Cabanis \& Heine, 1859) }\end{array}$ & $\begin{array}{l}\text { arapaçu-escamado-do- } \\
\text { sul }\end{array}$ & 10 & & FM & Jan, Abr-Jun, Set e Nov \\
\hline Dendrocolaptes platyrostris Spix, $1825^{*}$ & arapaçu-de-bico-preto & 62 & & FM FS CP GJ RA & Jan-Dez \\
\hline Xiphocolaptes albicollis (Vieillot, 1818)* & $\begin{array}{l}\text { cochi-de-garganta- } \\
\text { branca }\end{array}$ & 66 & & FM FS CP RA & Jan-Dez \\
\hline Furna & & & & & \\
\hline
\end{tabular}


Tabela 1. Continuação...

\begin{tabular}{|c|c|c|c|c|c|}
\hline Espécie & Nome popular & Contatos & Status & Fitofisionomias & Meses \\
\hline Xenops minutus (Sparrman, 1788)* & bico-virado-miúdo & 19 & & FM FS & Mar-Jul, Set, Nov e Dez \\
\hline Xenops rutilans Temminck, 1821 & bico-virado-carijó & 22 & & FM FS CP RA & Jan, Mar-Maio, Set-Dez \\
\hline Furnarius rufus (Gmelin, 1788)* & joão-de-barro & 4 & & GJ & Jan-Jun, Ago-Dez \\
\hline Lochmias nematura (Lichtenstein, 1823)* & joão-do-riacho & 89 & & FM FS CP (MR) & Jan-Dez \\
\hline Automolus leucophthalmus (Wied, 1821)* & $\begin{array}{l}\text { barranqueiro-de-olho- } \\
\text { branco }\end{array}$ & 171 & & FM FS CP RA & Jan-Dez \\
\hline Anabazenops fuscus (Vieillot, 1816)* & trepador-coleira & 76 & & FM FS CP (TA) & Fev-Dez \\
\hline Philydor lichtensteini Cabanis \& Heine, 1859 & limpa-folha-ocráceo & 20 & & FM FS & Fev-Set, Nov \\
\hline Philydor atricapillus (Wied, 1821)* & limpa-folha-coroado & 291 & & FM FS RA & Jan-Dez \\
\hline Philydor rufum (Vieillot, 1818)* & $\begin{array}{l}\text { limpa-folha-de-testa- } \\
\text { baia }\end{array}$ & 317 & & FM FS RA & Jan-Dez \\
\hline Heliobletus contaminatus Berlepsch, 1885* & trepadorzinho & 45 & & FM CP RA & Fev-Jun, Ago-Dez \\
\hline Anabacerthia amaurotis (Temminck, 1823) & limpa-folha-miúdo & 29 & & FM & Jan-Set e Nov \\
\hline Syndactyla rufosuperciliata (Lafresnaye, 1832) & limpa-folha-quiete & 5 & & FM (TA) & Maio e Out \\
\hline $\begin{array}{l}\text { Cichlocolaptes leucophrus } \\
\text { (Jardine \& Selby, 1830) }\end{array}$ & trepador-sobrancelha & 21 & & FM FS & Jan-Jul, Out-Dez \\
\hline Leptasthenura setaria (Temminck, 1824)* & grimpeiro & 6 & & RA & $\begin{array}{l}\text { Jan, Fev, Abr-Jun, Ago- } \\
\text { Dez }\end{array}$ \\
\hline Synallaxis ruficapilla Vieillot, 1819* & pichororé & 119 & & FM FS CP RA & Jan-Dez \\
\hline Synallaxis cinerascens Temminck, 1823 & pi-puí & 1 & & FM & Ago \\
\hline Synallaxis spixi Sclater, 1856* & joão-teneném & 19 & & CP GJ & $\begin{array}{l}\text { Fev, Mar, Maio, Jun, } \\
\text { Ago-Nov }\end{array}$ \\
\hline Cranioleuca pallida (Wied, 1831)* & joão-pálido & 32 & & FM CP GJ RA & $\begin{array}{l}\text { Mar, Abr, Maio, Jun, } \\
\text { Ago-Nov }\end{array}$ \\
\hline \multicolumn{6}{|l|}{ Pipridae } \\
\hline Neopelma chrysolophum Pinto, 1944* & fruxu & 3 & & FM CP & Abr e Out \\
\hline Manacus manacus (Linnaeus, 1766) & rendeira & 21 & & FS CP & Fev, Mar, Maio-Out \\
\hline Ilicura militaris (Shaw \& Nodder, 1809)* & tangarazinho & 53 & & FM FS CP GJ & Mar-Nov \\
\hline $\begin{array}{l}\text { Chiroxiphia caudata (Shaw \& Nodder, 1793)* } \\
\text { Tityridae }\end{array}$ & tangará-dançarino & 889 & & FM FS CP RA & Jan-Dez \\
\hline Oxyruncus cristatus Swainson, 1821* & bico-agudo & 37 & & FM FS & Jan-Dez \\
\hline Onychorhynchus swainsoni (Pelzeln, 1858) & maria-leque-do-sudeste & 2 & VU & FM (MR) & Maio e Set \\
\hline Myiobius barbatus (Gmelin, 1789) & assanhadinho & 19 & & FS & $\begin{array}{l}\text { Fev, Maio-Jul, Set, Out } \\
\text { e Dez }\end{array}$ \\
\hline Schiffornis virescens (Lafresnaye, 1838)* & flautim-verde & 378 & & FM FS CP RA & Jan-Dez \\
\hline Tityra inquisitor (Lichtenstein, 1823$)^{\mathrm{m}}$ & $\begin{array}{l}\text { araponguinha-de-cara- } \\
\text { preta }\end{array}$ & 19 & & FM FS CP GJ & Out-Abr \\
\hline Tityra cayana (Linnaeus, 1766$)^{\mathrm{m}}$ & $\begin{array}{l}\text { araponguinha-de-rabo- } \\
\text { preto }\end{array}$ & 36 & & FM FS CP GJ RA & Set-Mar \\
\hline Pachyramphus viridis (Vieillot, 1816) & caneleiro-verde & 1 & & FM & Dez \\
\hline $\begin{array}{l}\text { Pachyramphus castaneus } \\
\text { (Jardine \& Selby, 1827)* }\end{array}$ & caneleiro-castanho & 140 & & FM FS CP GJ RA & Jan-Dez \\
\hline Pachyramphus polychopterus (Vieillot, 1818)*m & caneleiro-preto & 21 & & FM FS CP RA & Set-Abr \\
\hline $\begin{array}{l}\text { Pachyramphus marginatus } \\
\text { (Lichtenstein, 1823)* }\end{array}$ & caneleiro-bordado & 16 & NT & FS & Fev, Mar, Maio-Dez \\
\hline $\begin{array}{l}\text { Pachyramphus validus (Lichtenstein, 1823)* m } \\
\text { Cotingidae }\end{array}$ & caneleiro-de-crista & 37 & & FM FS CP GJ RA & Set-Jan \\
\hline Lipaugus lanioides (Lesson, 1844)* & cricrió-suisso & 32 & VU & FM FS & Jan, Fev, Maio-Dez \\
\hline Procnias nudicollis (Vieillot, 1817)* & araponga & 188 & VU & FM FS CP RA & Jan-Fev, Abr-Dez \\
\hline Pyroderus scutatus (Shaw, 1792)* & pavó & 108 & VU & FM FS CP GJ RA & Jan-Dez \\
\hline Carpornis cucullata (Swainson, 1821)* & corocochó & 231 & & FM FS RA & Jan-Dez \\
\hline Carpornis melanocephala (Wied, 1820)* & cochó & 50 & VU & FS & Jan, Mar-Dez \\
\hline $\begin{array}{l}\text { Phibalura flavirostris Vieillot, } 1816 \\
\text { Tyrannoidea Incertae sedis }\end{array}$ & tesourinha-da-mata & 1 & NT & GJ & Abr \\
\hline Platyrinchus mystaceus Vieillot, 1818* & patinho & 284 & & FM FS CP RA & Jan-Dez \\
\hline Piprites chloris (Temminck, 1822)* & caneleirinho-cantor & 34 & & FM RA & Jan, Mar-Dez \\
\hline
\end{tabular}


Tabela 1. Continuação...

\begin{tabular}{|c|c|c|c|c|c|}
\hline Espécie & Nome popular & Contatos & Status & Fitofisionomias & Meses \\
\hline \multicolumn{6}{|l|}{ Rhynchocyclidae } \\
\hline Mionectes rufiventris Cabanis, 1846* & $\begin{array}{l}\text { abre-asa-de-cabeça- } \\
\text { cinza }\end{array}$ & 141 & & FM FS CP GJ RA & Jan-Dez \\
\hline Leptopogon amaurocephalus Tschudi, 1846* & abre-asa-cabeçudo & 248 & & FM FS CP RA & Jan-Dez \\
\hline Phylloscartes ventralis (Temminck, 1824)* & maria-borboleta & 12 & & FM RA & Fev, Abr-Jun, Out \\
\hline Phylloscartes paulista Ihering \& Ihering, 1907* & não-pode-parar & 13 & VU & FS & $\begin{array}{l}\text { Abr, Jun, Jul, Set, Out } \\
\text { e Dez }\end{array}$ \\
\hline Phylloscartes oustaleti (Sclater, 1887)* & treme-rabo & 324 & & FM FS RA & Jan-Dez \\
\hline $\begin{array}{l}\text { Phylloscartes sylviolus } \\
\text { (Cabanis \& Heine, 1859)* }^{\text {(Canis }}\end{array}$ & maria-pequena & 42 & NT & FS & Jan-Ago \\
\hline Tolmomyias sulphurescens (Spix, 1825)* & $\begin{array}{l}\text { bico-chato-de-orelha- } \\
\text { preta }\end{array}$ & 230 & & FM FS CP GJ RA & Jan-Dez \\
\hline Todirostrum poliocephalum (Wied, 1831)* & ferreirinho-teque-teque & 67 & & FM FS CP & Jan-Dez \\
\hline Poecilotriccus plumbeiceps (Lafresnaye, 1846)* & tororó & 31 & & $\mathrm{CP}$ & Fev, Mar-Jun \\
\hline Myiornis auricularis (Vieillot, 1818)* & maria-cigarra & 302 & & FM FS CP RA & Jan-Dez \\
\hline Hemitriccus diops (Temminck, 1822) & maria-olho-falso & 1 & & FM (TA) & Maio \\
\hline $\begin{array}{l}\text { Hemitriccus obsoletus (Miranda-Ribeiro, } \\
\text { 1906)* }\end{array}$ & maria-catraca & 7 & & FM (TA) & Set-Nov \\
\hline Hemitriccus orbitatus (Wied, 1831) & maria-tiririzinha & 21 & & FS & Fev-Dez \\
\hline Hemitriccus nidipendulus (Wied, 1831) & maria-verdinha & 1 & & $\mathrm{CP}$ & Maio \\
\hline \multicolumn{6}{|l|}{ Tyrannidae } \\
\hline $\begin{array}{l}\text { Tyranniscus burmeisteri (Cabanis \& Heine, } \\
\text { 1859)* }\end{array}$ & piolhinho-chiador & 14 & & FM FS & Abr-Jun, Ago-Nov \\
\hline Camptostoma obsoletum (Temminck, 1824)* & risadinha & 27 & & FM CP & Jan, Fev, Abr-Dez \\
\hline Elaenia flavogaster (Thunberg, 1822)* & maria-é-dia & 11 & & CP GJ & Fev, Abr, Out-Dez \\
\hline Elaenia chilensis Hellmayr, 1927 & $\begin{array}{l}\text { guaracava-de-crista- } \\
\text { branca }\end{array}$ & 1 & & $\mathrm{CP}$ & Abr \\
\hline Elaenia parvirostris Pelzeln, 1868 & guaracava-de-bico-curto & 3 & & $\mathrm{CP}$ & Abr e Nov \\
\hline Elaenia mesoleuca (Deppe, 1830) & tuque & 2 & & GJ & Nov \\
\hline Myiopagis caniceps (Swainson, 1835)* & maria-da-copa & 53 & & FS & Jan, Mar-Jul, Set-Dez \\
\hline Phyllomyias virescens (Temminck, 1824) & piolhinho-verdoso & 2 & & $\mathrm{CP}$ & Abr e Jun \\
\hline Phyllomyias fasciatus (Thunberg, 1822)* & piolhinho & 17 & & CP GJ & Jan, Abr, Jun-Out, Dez \\
\hline Phyllomyias griseocapilla Sclater, 1862* & piolhinho-serrano & 41 & & FM FS & Mar-Set, Nov e Dez \\
\hline Serpophaga subcristata (Vieillot, 1817)* & alegrinho & 24 & & CP GJ & Jan, Abr-Dez \\
\hline Attila phoenicurus Pelzeln, 1868* m & tinguaçu-castanho & 120 & & FM FS CP RA & Set-Mar \\
\hline Attila rufus (Vieillot, 1819)* & capitão-de-saíra & 231 & & FM FS CP GJ RA & Jan-Dez \\
\hline Legatus leucophaius (Vieillot, 1818)* m & bem-te-vi-pirata & 36 & & FM FS CP GJ & Set-Fev \\
\hline $\begin{array}{l}\text { Ramphotrigon megacephalum (Swainson, } \\
1835)^{*}\end{array}$ & maria-cabeçuda & 7 & & FM FS (TA) & Fev, Jul, Ago, Out-Dez \\
\hline Myiarchus swainsoni Cabanis \& Heine, 1859* m & irré & 122 & & FM FS CP GJ RA & Set-Mar \\
\hline Sirystes sibilator (Vieillot, 1818)* & maria-assobiadeira & 22 & & FM FS & $\begin{array}{l}\text { Fev, Mar, Maio-Set, } \\
\text { Nov e Dez }\end{array}$ \\
\hline Pitangus sulphuratus (Linnaeus, 1766)* & bem-te-vi-verdadeiro & 104 & & CP GJ & Jan, Fev, Abr-Dez \\
\hline Machetornis rixosa (Vieillot, 1819)* & suiriri-cavaleiro & 4 & & GJ & \\
\hline $\begin{array}{l}\text { Myiodynastes maculatus (Statius Muller, } \\
1776)^{*} \mathrm{~m}\end{array}$ & bem-te-vi-rajado & 146 & & FM FS CP GJ RA & Set-Fev \\
\hline Megarynchus pitangua (Linnaeus, 1766)* & neinei & 52 & & FM FS CP GJ RA & \\
\hline Myiozetetes similis (Spix, 1825)* & $\begin{array}{l}\text { bem-te-vi-de-coroa- } \\
\text { vermelha }\end{array}$ & 75 & & FM FS CP GJ & Jan-Dez \\
\hline Tyrannus melancholicus Vieillot, 1819* m & suiriri & 13 & & CP GJ RA & Set-Abr \\
\hline Tyrannus savana Vieillot, 1808 & tesourinha-do-campo & 1 & & GJ & Dez \\
\hline Empidonomus varius (Vieillot, 1818) ${ }^{\mathrm{m}}$ & peitica & 18 & & FM FS CP GJ RA & Set-Fev \\
\hline Conopias trivirgatus (Wied, 1831)* & bem-te-vi-de-três-riscas & 36 & & FM FS CP & Mar, Jun, Ago-Dez \\
\hline Colonia colonus (Vieillot, 1818)* & maria-viuvinha & 40 & & FM FS CP & Fev-Jun, Ago-Dez \\
\hline Myiophobus fasciatus (Statius Muller, 1776)* & felipe & 3 & & $\mathrm{CP}$ & Nov \\
\hline Pyrocephalus rubinus (Boddaert, 1783) ${ }^{\mathrm{m}}$ & princípe & 4 & & GJ & Maio, Jun, Ago e Out \\
\hline
\end{tabular}


Tabela 1. Continuação...

\begin{tabular}{|c|c|c|c|c|c|}
\hline Espécie & Nome popular & Contatos & Status & Fitofisionomias & Meses \\
\hline Fluvicola nengeta (Linnaeus, 1766) & lavadeira-mascarada & 1 & & AB GJ & Dez \\
\hline Lathrotriccus euleri (Cabanis, 1868)*m & enferrujado & 113 & & FM FS CP RA & Set-Abr \\
\hline Contopus cinereus (Spix, 1825) & piuí-cinza & 1 & & FM & Ago \\
\hline Knipolegus cyanirostris (Vieillot, 1818) & $\begin{array}{l}\text { maria-preta-de-bico- } \\
\text { azulado }\end{array}$ & 1 & & $\mathrm{CP}$ & Out \\
\hline Muscipipra vetula (Lichtenstein, 1823) & tesoura-cinza & 10 & & FM CP & Maio, Ago, Set-Nov \\
\hline \multicolumn{6}{|l|}{ Vireonidae } \\
\hline Cyclarhis gujanensis (Gmelin, 1789)* & pitiguari & 152 & & FM FS CP GJ RA & Jan-Dez \\
\hline Vireo olivaceus (Linnaeus, 1766)* m & juruviara & 155 & & FM FS CP GJ RA & Set-Abr \\
\hline Hylophilus poicilotis Temminck, 1822* & vite-vite-coroado & 100 & & FM CP RA & Jan-Dez \\
\hline \multicolumn{6}{|l|}{ Corvidae } \\
\hline Cyanocorax caeruleus (Vieillot, 1818)* & gralha-azul & 37 & NT & FS CP & Fev-Jul, Set-Nov \\
\hline \multicolumn{6}{|l|}{ Hirundinidae } \\
\hline Pygochelidon cyanoleuca (Vieillot, 1817)* & $\begin{array}{l}\text { andorinha-azul-e- } \\
\text { branca }\end{array}$ & 445 & & GJ CP (SO) & Jan-Dez \\
\hline Stelgidopteryx ruficollis (Vieillot, 1817)* & andorinha-serradora & 85 & & $\begin{array}{l}\text { FS GJ CP (SO) } \\
\text { (MR) }\end{array}$ & Jan-Maio, Ago-Dez \\
\hline Progne chalybea (Gmelin, 1789)* & andorinha-grande & 319 & & GJ CP (SO) & Jan-Dez \\
\hline Tachycineta leucorrhoa (Vieillot, 1817) & $\begin{array}{l}\text { andorinha-de-frente- } \\
\text { branca }\end{array}$ & 7 & & GJ & Set-Nov \\
\hline \multicolumn{6}{|l|}{ Troglodytidae } \\
\hline Troglodytes musculus Naumann, 1823* & corruíra-de-casa & 87 & & CP GJ & Jan-Dez \\
\hline Cantorchilus longirostris (Vieillot, 1819) & garrincha-trovão & 4 & & $\mathrm{CP}$ & Mar, Maio, Ago \\
\hline \multicolumn{6}{|l|}{ Polioptilidae } \\
\hline Ramphocaenus melanurus Vieillot, 1819* & bico-assovelado & 10 & & FM FS CP & Mar, Jul-Set, Nov e Dez \\
\hline \multicolumn{6}{|l|}{ Turdidae } \\
\hline Turdus flavipes Vieillot, 1818* & sabiá-una & 301 & & FM FS CP GJ RA & Jan-Abr, Jun-Dez \\
\hline Turdus rufiventris Vieillot, 1818* & sabiá-laranjeira & 81 & & FM FS CP GJ RA & Jan, Fev, Abr-Dez \\
\hline Turdus leucomelas Vieillot, 1818* & sabiá-de-cabeça-cinza & 33 & & GJ & $\begin{array}{l}\text { Jan, Fev, Abr-Jun, Ago- } \\
\text { Dez }\end{array}$ \\
\hline Turdus amaurochalinus Cabanis, 1850 & sabiá-poca & 20 & & FM FS CP GJ & Jan, Maio, Ago-Dez \\
\hline Turdus subalaris (Seebohm, 1887) & sabiá-ferreiro & 1 & & $\mathrm{CP}$ & Out \\
\hline Turdus albicollis Vieillot, 1818* & sabiá-coleira & 536 & & FM FS CP RA & Jan-Dez \\
\hline \multicolumn{6}{|l|}{ Mimidae } \\
\hline Mimus saturninus (Lichtenstein, 1823) & sabiá-do-campo & 1 & & GJ & Set \\
\hline \multicolumn{6}{|l|}{ Coerebidae } \\
\hline Coereba flaveola (Linnaeus, 1758)* & cambacica & 117 & & FM FS CP GJ RA & Jan-Dez \\
\hline \multicolumn{6}{|l|}{ Thraupidae } \\
\hline Saltator fuliginosus (Daudin, 1800)* & bico-de-pimenta & 119 & & FM FS CP & Jan-Dez \\
\hline Saltator similis d'Orbigny \& Lafresnaye, 1837* & $\begin{array}{l}\text { trinca-ferro-de-asa- } \\
\text { verde }\end{array}$ & 26 & & FM FS CP GJ RA & Jan, Mar, Jun, Ago-Dez \\
\hline Orchesticus abeillei (Lesson, 1839) & tiê-pardo & 9 & & FM FS & Abr, Ago-Nov \\
\hline Orthogonys chloricterus (Vieillot, 1819)* & catirumbava & 408 & & FM FS GJ CP & Fev-Dez \\
\hline Pyrrhocoma ruficeps (Strickland, 1844) & cabecinha-castanha & 2 & NT & FS & Set \\
\hline Tachyphonus coronatus (Vieillot, 1822)* & gurundi & 218 & & FM FS CP GJ RA & Jan-Dez \\
\hline Ramphocelus bresilius (Linnaeus, 1766) & tiê-sangue & 52 & & FS CP GJ & Jan-Dez \\
\hline Lanio cristatus (Linnaeus, 1766)* & tiê-galo & 166 & & FS CP GJ & Jan, Mar-Set, Nov-Dez \\
\hline Lanio melanops (Vieillot, 1818)* & tiê-de-topete & 273 & & FM FS CP GJ RA & Jan-Dez \\
\hline Tangara seledon (Statius Muller, 1776)* & saíra-de-sete-cores & 1274 & & FM FS CP GJ RA & Jan-Dez \\
\hline Tangara cyanocephala (Statius Muller, 1776)* & saíra-militar & 877 & & FM FS CP GJ RA & Jan-Dez \\
\hline Tangara desmaresti (Vieillot, 1819) & saíra-da-serra & 65 & & FM CP GJ RA & $\begin{array}{l}\text { Jan, Mar, Maio, Jun, Set, } \\
\text { Out e Dez }\end{array}$ \\
\hline Tangara sayaca (Linnaeus, 1766)* & sanhaço-cinza & 156 & & FM FS CP GJ RA & Jan-Dez \\
\hline Tangara cyanoptera (Vieillot, 1817)* & sanhaço-da-serra & 86 & & FM FS CP GJ RA & Jan, Fev, Abr-Dez \\
\hline Tangara palmarum (Wied, 1823)* & sanhaço-do-coqueiro & 95 & & FM FS CP GJ & Jan-Dez \\
\hline
\end{tabular}


Tabela 1. Continuação...

\begin{tabular}{|c|c|c|c|c|c|}
\hline Espécie & Nome popular & Contatos & Status & Fitofisionomias & Meses \\
\hline Tangara ornata (Sparrman, 1789)* & sanhaço-rei & 86 & & FM FS CP GJ RA & Jan-Dez \\
\hline Tangara cayana (Linnaeus, 1766)* & saíra-cabocla & 26 & & CP GJ & Fev, Abr, Jun, Out e Nov \\
\hline Stephanophorus diadematus (Temminck, 1823) & sanhaço-frade & 1 & & $\mathrm{CP}$ & Out \\
\hline Cissopis leverianus (Gmelin, 1788)* & tietinga & 48 & NT & FM FS CP & $\begin{array}{l}\text { Fev, Abr-Jul, Set, Nov } \\
\text { e Dez }\end{array}$ \\
\hline Pipraeidea melanonota (Vieillot, 1819)* & saíra-viúva & 16 & & FM CP GJ RA & Jan, Jun, Ago-Nov \\
\hline Tersina viridis (Illiger, 1811)* & saí-andorinha & 69 & & FM FS CP GJ RA & Fev-Jun, Ago-Dez \\
\hline Dacnis cayana (Linnaeus, 1766) & saí-azul & 77 & & FM FS CP GJ RA & Jan-Nov \\
\hline Chlorophanes spiza (Linnaeus, 1758)* & saí-verde & 11 & & FS GJ & Fev, Abr, Jul-Set \\
\hline Hemithraupis ruficapilla (Vieillot, 1818)* & saíra-ferrugem & 364 & & FM FS CP GJ RA & Jan-Dez \\
\hline \multicolumn{6}{|l|}{ Emberizidae } \\
\hline Zonotrichia capensis (Statius Muller, 1776)* & tico-tico-verdadeiro & 42 & & GJ & Jan-Dez \\
\hline Ammodramus humeralis (Bosc, 1792) & tico-tico-do-campo & 2 & & GJ & Set e Nov \\
\hline Haplospiza unicolor Cabanis, $1851^{*}$ & catatau & 58 & & FM FS CP (TA) & Fev-Jun, Ago-Dez \\
\hline Sicalis flaveola (Linnaeus, 1766)* & canário-da-terra & 54 & & GJ & Jan-Dez \\
\hline Volatinia jacarina (Linnaeus, 1766) & tiziu & 25 & & GJ & Jan, Fev, Jun, Set-Dez \\
\hline Sporophila frontalis (Verreaux, 1869)* & pichochó & 30 & EN & FM FS CP (TA) & $\begin{array}{l}\text { Jan-Jun, Ago, Set, Nov- } \\
\text { Dez }\end{array}$ \\
\hline Sporophila falcirostris (Temminck, 1820)* & papa-capim-da-taquara & 32 & $\mathrm{EN}$ & FM FS CP (TA) & $\begin{array}{l}\text { Mar, Maio-Set, Nov } \\
\text { e Dez }\end{array}$ \\
\hline Sporophila lineola $(\text { Linnaeus, } 1758)^{\mathrm{m}}$ & bigodinho & 12 & & GJ & Nov-Fev \\
\hline Sporophila caerulescens (Vieillot, 1823)* & coleirinha & 33 & & GJ & Jan-Maio, Out-Dez \\
\hline Tiaris fuliginosus (Wied, 1830) & cigarrinha-do-coqueiro & 1 & & FS (TA) & Dez \\
\hline Arremon semitorquatus Swainson, 1838 & $\begin{array}{l}\text { tico-tico-de-coleira- } \\
\text { falha }\end{array}$ & 2 & & FS & Maio e Out \\
\hline \multicolumn{6}{|l|}{ Cardinalidae } \\
\hline Habia rubica (Vieillot, 1817)* & tiê-da-mata & 905 & & FM FS RA & Jan-Dez \\
\hline \multicolumn{6}{|l|}{ Parulidae } \\
\hline Parula pitiayumi (Vieillot, 1817)* & mariquita & 71 & & FM FS CP GJ RA & Jan-Dez \\
\hline Geothlypis aequinoctialis (Gmelin, 1789)* & pia-cobra & 6 & & $\mathrm{AB}$ & Jan, Abr, Jun, Ago, Out \\
\hline Basileuterus culicivorus (Deppe, 1830)* & pula-pula-coroado & 786 & & FM FS CP GJ RA & Jan-Dez \\
\hline Basileuterus hypoleucus Bonaparte, 1830 & pula-pula-pichito & 1 & & RA & Maio \\
\hline Basileuterus leucoblepharus (Vieillot, 1817)* & pula-pula-assobiador & 34 & & FM CP RA & Jan-Dez \\
\hline Phaeothlypis rivularis (Wied, 1821)* & pula-pula-ribeirinho & 391 & & FM FS CP (MR) & Jan-Dez \\
\hline \multicolumn{6}{|l|}{ Icteridae } \\
\hline Psarocolius decumanus (Pallas, 1769) & japu-preto & 3 & & GJ & Ago \\
\hline Cacicus chrysopterus (Vigors, 1825)* & soldado & 109 & & FM CP GJ RA & Jan-Jun, Ago-Dez \\
\hline Cacicus haemorrhous (Linnaeus, 1766)* & guaxe & 460 & & FM FS CP & Jan-Dez \\
\hline Icterus pyrrhopterus (Vieillot, 1819)* & encontro & 3 & & $\mathrm{CP}$ & Out \\
\hline Molothrus bonariensis (Gmelin, 1789) & chopim & 17 & & GJ & Maio-Dez \\
\hline \multicolumn{6}{|l|}{ Fringillidae } \\
\hline Sporagra magellanica (Vieillot, 1805)* & $\begin{array}{l}\text { pintassilgo-de-cabeça- } \\
\text { preta }\end{array}$ & 4 & & GJ & Maio e Out \\
\hline Euphonia chlorotica (Linnaeus, 1766) & vi-vi & 2 & & $\mathrm{CP}$ & Jan e Jun \\
\hline Euphonia violacea (Linnaeus, 1758)* & gaturamo-verdadeiro & 50 & & FM FS CP GJ & Jan-Dez \\
\hline Euphonia chalybea (Mikan, 1825) & cais-cais & 8 & VU & FM GJ & Maio, Set e Nov \\
\hline Euphonia cyanocephala (Vieillot, 1818) & gaturamo-rei & 2 & & GJ & Dez \\
\hline Euphonia pectoralis (Latham, 1801)* & ferro-velho & 335 & & FM FS CP GJ RA & Jan-Dez \\
\hline Chlorophonia cyanea (Thunberg, 1822) & gaturamo-bandeira & 1 & & FS & Jul \\
\hline
\end{tabular}

logísticas, porém, como observa-se na Figura 1, as fitofisionomias mais representativas já foram contempladas. A riqueza e a composição das espécies são similares às encontradas para o contíguo Parque Estadual Intervales (Vielliard \& Silva 2001, Aleixo \& Galetti 1997, Willis \& Oniki 2003).
Espécies colonizadoras, vagantes e localmente raras poderão continuar a ser encontradas por um período de tempo imprevisível, como se observa ao se analisar os levantamentos de áreas amostradas por mais de cinquenta anos (Magalhães 1999, Cavarzere et al. 2010). A ausência de estabilização da curva do coletor, a estimativa obtida 


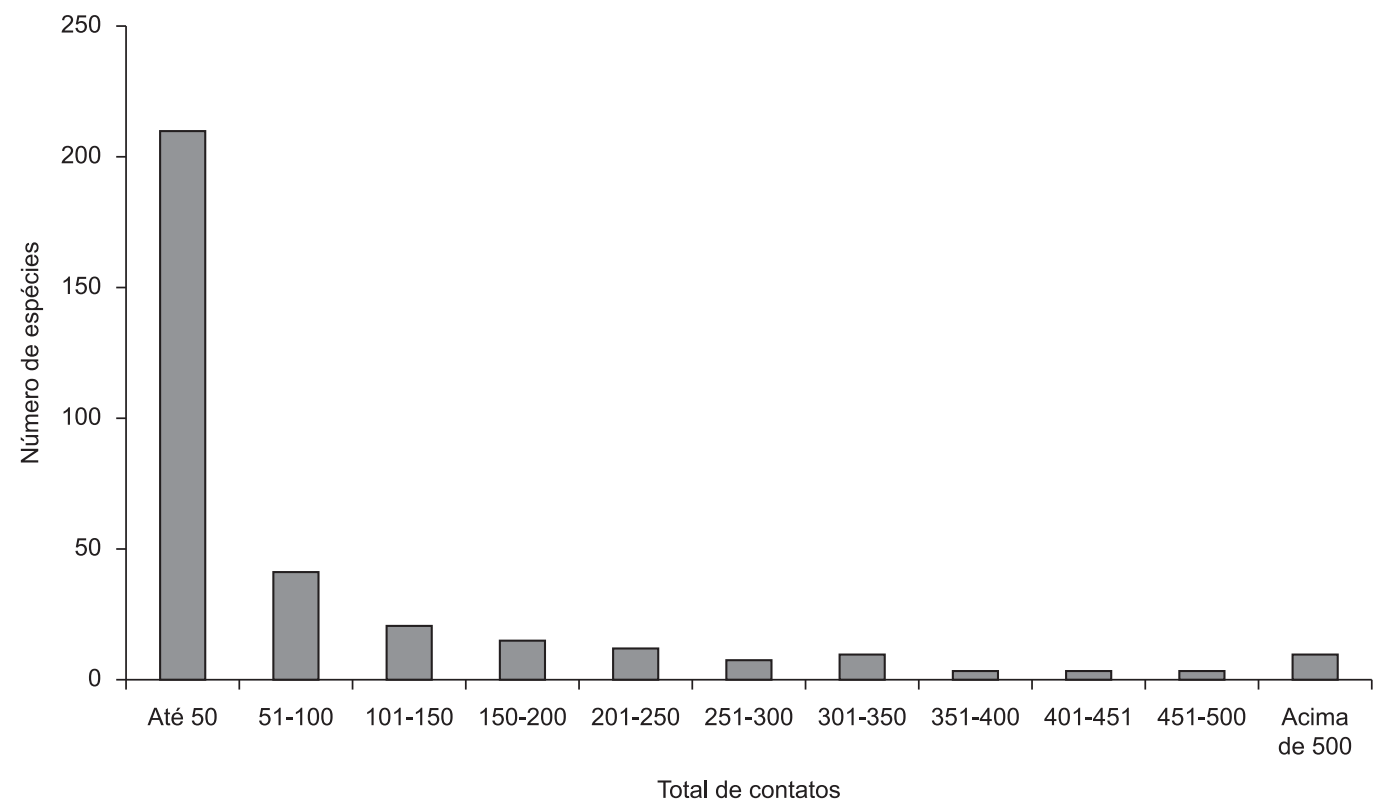

Figura 2. Distribuição das espécies de aves registradas no Parque Estadual Carlos Botelho em classes de total de contatos.

Figure 2. Bird species distribution in total contacts classes for Carlos Botelho State Park, São Paulo southeastern Brazil.

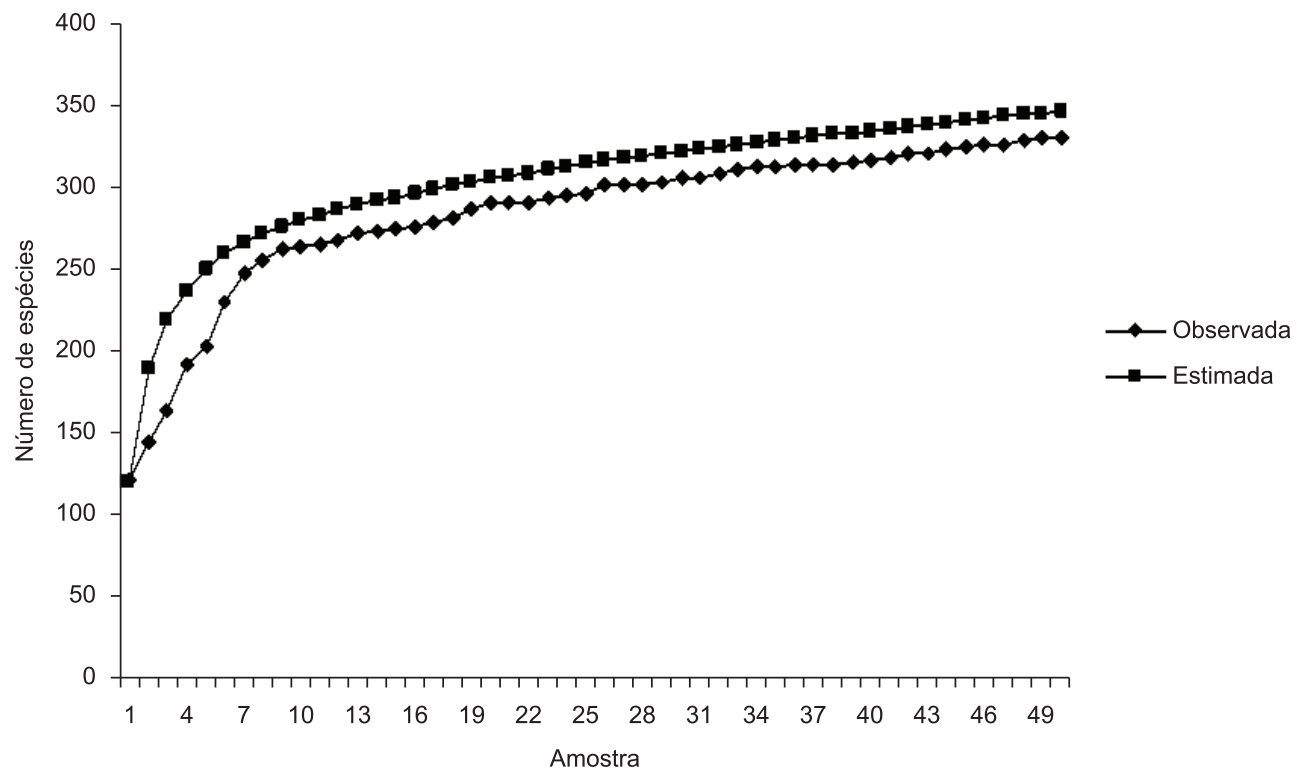

Figura 3. Curvas de acúmulo de espécies de aves observada e estimada (método Bootstrap) para o período amostral de abril de 2006 a dezembro de 2009 no Parque Estadual Carlos Botelho.

Figure 3. Observed and estimated (Bootstrap) bird species accumulation curves between April 2006- December 2009 for Carlos Botelho State Park.

pelo Bootstrap e o registro de espécies por outros pesquisadores ou no entorno do parque, apóiam essa constatação. A caracterização da chamada avifauna nuclear é um objetivo mais factível de ser atingido do que um inventário completo (Remsen 1994, Cohn-Haft et al. 1997). No caso do PECB as espécies florestais, residentes e migratórias, constituem essa avifauna nuclear. O emprego de outras metodologias de amostragem como capturas com redes de neblina, uso de playbacks e observações focais em fruteiras e flores, provavelmente pode contribuir para atingir tal objetivo (Remsen 1994).

O período de setembro a novembro corresponde ao início da estação reprodutiva para a maior parte das espécies de aves no sudeste do Brasil (Sick 1997), o que pode ter colaborado para os valores mais elevados de riqueza mensal encontrados. As espécies classificadas como provavelmente migratórias localmente, também são consideradas migratórias na maior parte do estado de São Paulo (Willis \& Oniki 2003). Para outras espécies, relacionadas como migratórias no estado por Willis \& Oniki (2003), foram obtidos poucos registros, não permitindo definir se tratavam-se de migrantes ou vagantes. Adicionalmente, é provável a ocorrência de migrações altitudinais no interior do PECB, principalmente envolvendo espécies frugívoras (Castro et al. 2012). 


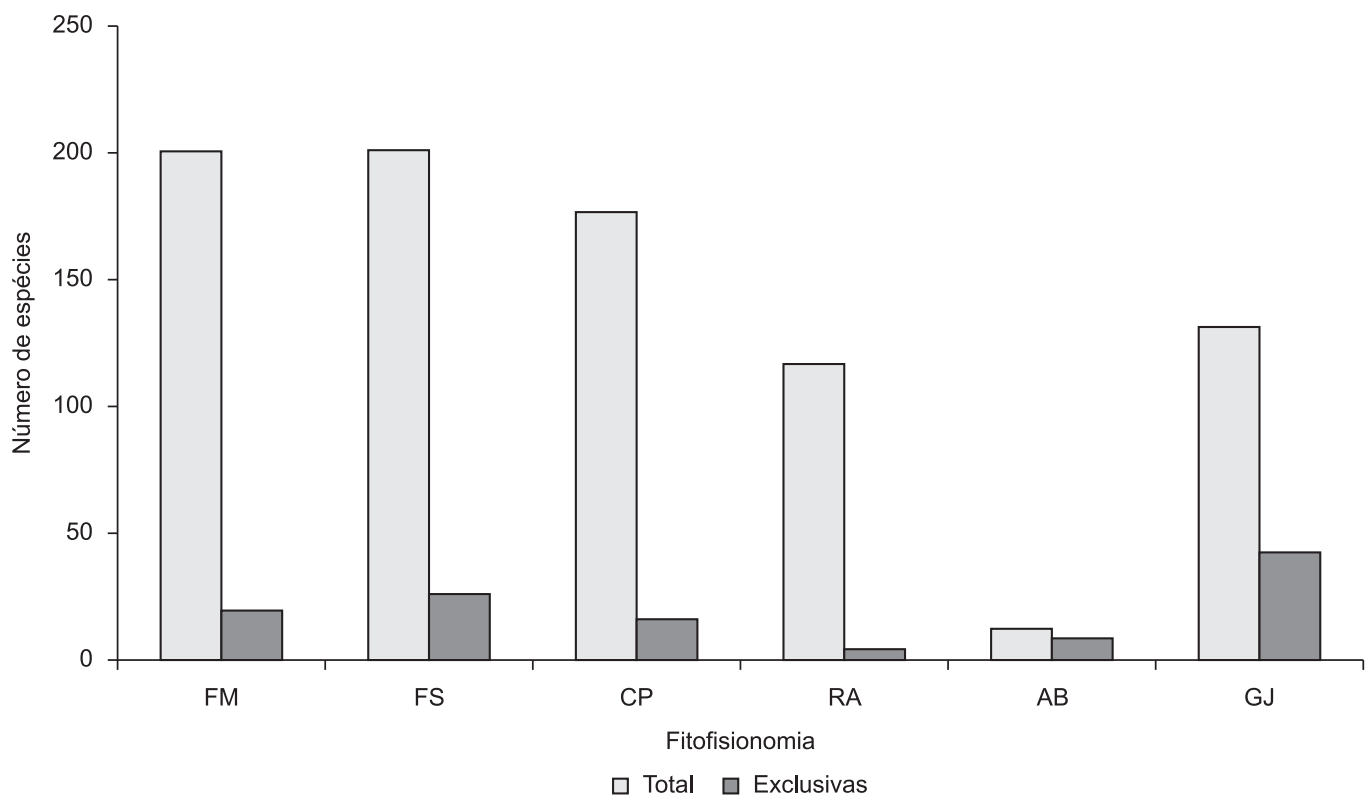

Figura 4. Distribuição das espécies de aves nas fitofisionomias amostradas no Parque Estadual Carlos Botelho. Espécies exclusivas são aquelas que foram encontradas num único tipo de vegetação durante o período amostral. $\mathrm{AB}=$ açudes e brejos; $\mathrm{CP}=$ capoeiras; $\mathrm{GJ}=$ gramados e jardins; $\mathrm{FM}=\mathrm{Floresta}$ Ombrófila Densa Montana; FS = Floresta Ombrófila Densa Submontana e RA = reflorestamento com pinheiros-do-Paraná.

Figure 4. Bird species distribution in Carlos Botelho State Park in sampled vegetation types. Exclusive species are recorded only in one vegetation type. $\mathrm{AB}=$ ponds and swamps; $\mathrm{CP}=$ secondary forest $\mathrm{GJ}=$ lawns and gardens; $\mathrm{FM}=$ lower highlands evergreen tropical forest; $\mathrm{FS}=$ lower plains evergreen tropical forest and $\mathrm{RA}=$ Parana pine reforestation.

Tabela 2. Valores médios de riqueza e total de contatos, acompanhados pelos respectivos desvios padrões, para a avifauna do Parque Estadual Carlos Botelho entre abril de 2006 e dezembro de 2009.

Table 2. Mean values of richness and total contacts, accompanied by standard deviations, for avifauna from Carlos Botelho State Park between April 2006 and December 2009.

\begin{tabular}{lcc}
\hline \multicolumn{1}{c}{ Mês (n) } & Riqueza Média & $\begin{array}{c}\text { Média do total de } \\
\text { contatos }\end{array}$ \\
\hline Janeiro (4) & $125 \pm 12,49$ & $592 \pm 115,83$ \\
Fevereiro (3) & $136 \pm 5$ & $749 \pm 218,11$ \\
Março (3) & $123 \pm 15,59$ & $812 \pm 116,09$ \\
Abril (5) & $136,25 \pm 7,32$ & $713,75 \pm 84,17$ \\
Maio (4) & $122,50 \pm 13,62$ & $756,5 \pm 234,62$ \\
Junho (5) & $124,75 \pm 6,75$ & $839,75 \pm 223,76$ \\
Julho (4) & $112,75 \pm 26,38$ & $661 \pm 352,91$ \\
Agosto (4) & $122,33 \pm 11,37$ & $706 \pm 162,37$ \\
Setembro (6) & $151,75 \pm 37,74$ & $1057,5 \pm 499,08$ \\
Outubro (4) & $144,50 \pm 9,81$ & $602,25 \pm 156,33$ \\
Novembro (4) & $146,25 \pm 18,28$ & $700,75 \pm 172,00$ \\
Dezembro (4) & $137,75 \pm 8,26$ & $684,75 \pm 86,73$ \\
\hline
\end{tabular}

As fitofisionomias antropogênicas, que ocupam áreas restritas no interior do parque, apresentaram espécies registradas exclusivamente em seus habitats e que em geral foram pouco contatadas. Além de espécies residentes que provavelmente apresentam populações pequenas, nesse conjunto estão incluídas vagantes e ocasionais. De maneira similar ao observado em outras localidades do sudeste do Brasil (Willlis \& Oniki 2002), as aves que ocupam essas fitofisionomias são em geral de menor preocupação conservacionista. Na região serrana do Espírito Santo onde os remanescentes florestais avaliados estão numa matriz agrícola, os percentuais de espécies registradas exclusivamente em habitats antropogênicos variaram entre 25 e 30\% da avifauna local, dependendo do grau de degradação das áreas (Willis \& Oniki 2002). Tais valores são similares ao registrado para o PECB. A presença da maioria dessas espécies no parque parece estar condicionada ao uso da terra nas propriedades do entorno. Recentemente, áreas com pastagens vizinhas à sede do PECB foram reflorestadas com eucaliptos, o que poderá ter impacto nas populações locais de algumas espécies no futuro.

Em relação às espécies florestais registradas em habitats antropogênicos, pode ser que essas áreas estejam absorvendo excedentes populacionais das florestas contíguas no caso de formas mais generalistas, que estejam funcionando como áreas de "dreno" para populações de algumas destas, ou que para certas espécies ocorra apenas o uso ocasional, ligado à disponibilidade de determinados recursos (Harris \& Pimm 2004, Barlow et al. 2010). Essas possibilidades precisam ser investigadas, pois essa maior flexibilidade no uso de habitats, apresentada por grande parte das aves locais, não necessariamente favorecerá suas populações em casos de perda ou degradação da floresta (Aleixo 2001).

O grimpeiro Leptasthenura setaria (Temminck, 1824) destacase entre as espécies exclusivas de habitats antropogênicos, por ser dependente dos pinheiros-do-paraná e estar expandido sua área de distribuição, colonizando plantios dessa árvore localizados próximos a áreas de ocorrência natural da fitofisionomia Floresta Ombrófila Mista (Antunes et al. 2007, Cabanne et al. 2007).

Diferenças locais nas assembleias de aves florestais foram detectadas em pesquisas que abordaram a influência da vegetação sobre a avifauna, em escalas mais refinadas do que a utilizada no presente estudo. Antunes et al. (2011) indicaram que os estádios médio e avançado de sucessão ecológica das florestas montanas e submontanas do PECB não diferem quanto à riqueza de espécies, mas sim em relação à composição das assembleias, com números similares de espécies exclusivas a cada uma delas. Já trechos da 


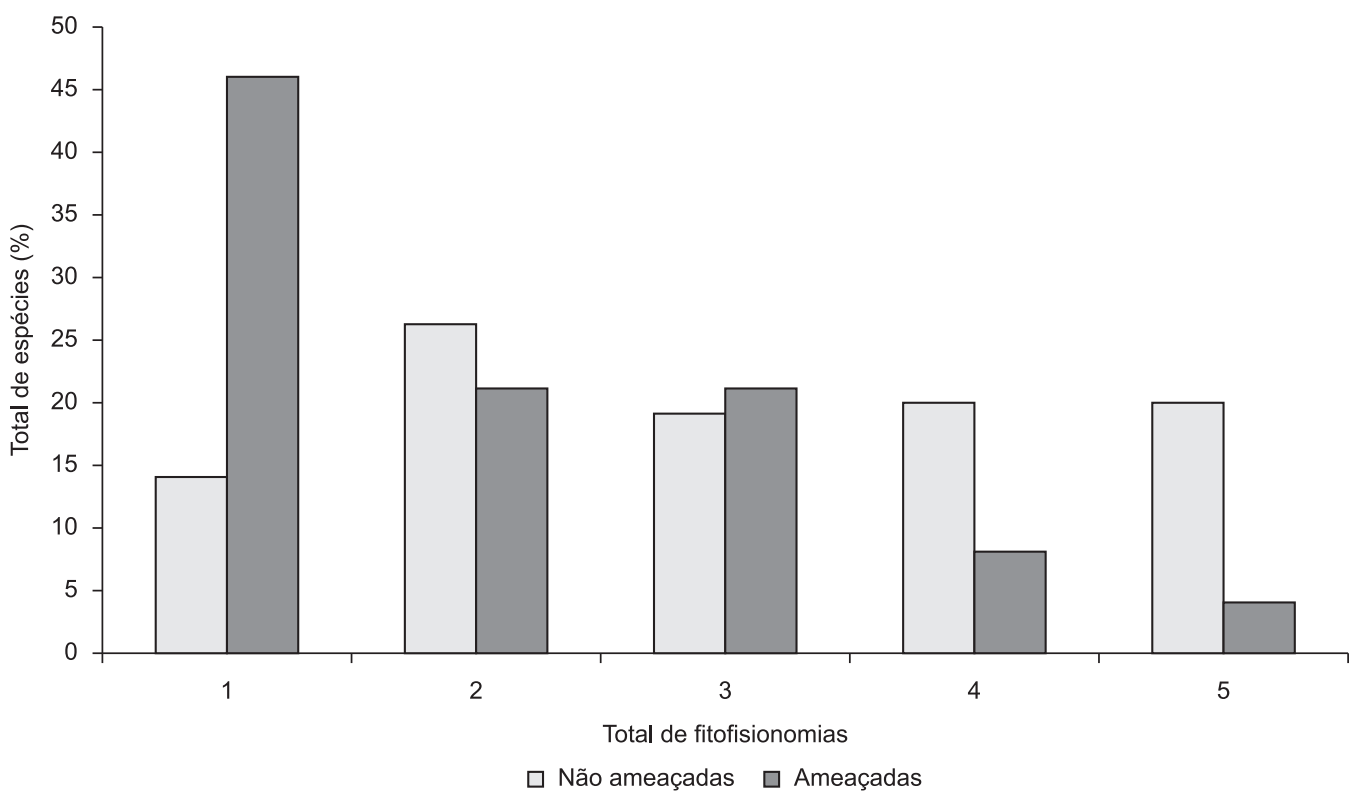

Figura 5. Distribuição percentual de espécies das aves do Parque Estadual Carlos Botelho, ameaçadas e de menor preocupação conservacionista, em relação ao número de fitofisionomias em que foram registradas.

Figure 5. Percentage distribution of threatened and least concern birds in Carlos Botelho State Park in relation to vegetation types number that were recorded.

Floresta Montana nos estádios inicial e médio apresentaram maior heterogeneidade estrutural da vegetação, resultando em maior riqueza e maior número de espécies exclusivas de aves, do que trechos no estádio avançado dessa fitofisionomia (Silva et al. 2010).

O principal vetor de impacto à biota do parque atualmente é a exploração ilegal do palmito da palmeira-juçara, que gera a degradação da floresta, a perda de recurso alimentar para frugívoros e a caça de espécies cinegéticas (Galetti \& Aleixo 1997). Historicamente esta atividade está concentrada em certos trechos mais vulneráveis, como as divisas e as vias de acesso originadas a partir da estrada estadual (SP 139) que corta o parque. Durante o período de estudo, constatou-se que esteve constantemente sujeita às ações repressivas dos guarda-parques e policiais ambientais. Projetos para a geração de renda e educação ambiental para os moradores do entorno têm sido efetuados e espera-se que resultem no declínio da atividade (São Paulo 2008a). A estrada é outro vetor de impacto importante, além de atropelamentos, ela serve de via de acesso para infratores ambientais, carreia lixo, poluentes e sedimentos para trechos da floresta e dos cursos d' água, e contribui para o estabelecimento de espécies vegetais exóticas invasoras (São Paulo 2008a). Além disso, descontinuidades da cobertura florestal, como estradas e linhas de transmissão de energia elétrica, prejudicam ou mesmo inviabilizam o deslocamento de certas espécies entre blocos de vegetação por elas seccionados. Esse efeito de barreira da rodovia foi detectado para a tovaca Chamaeza campanisona (Lichtenstein, 1823) (Oliveira et al. 2011).

Espera-se que o panorama elaborado sobre a avifauna do PECB, baseado em dados qualitativos e quantitativos obtidos periodicamente por um período de quase quatro anos, possa futuramente contribuir para se avaliar padrões de diversidade local no tempo e no espaço. A partir da lista de aves consolidada podem ser selecionados táxons ou grupos funcionais apropriados para subsidiar protocolos de monitoramento ambiental (ver Silva et al. 2010, Oliveira et al. 2011). O conhecimento gerado sobre a avifauna do PECB também poderá contribuir com informações para os programas de educação ambiental e ecoturismo, que em conjunto com a preservação dos ecossistemas e a realização de pesquisas científicas constituem os objetivos de um Parque Estadual (Brasil 2000).

\section{Agradecimentos}

Ao diretor do Parque Estadual Carlos Botelho, Engenheiro José Luiz Camargo Maia pelo apoio à pesquisa. Aos revisores anônimos pelas críticas e sugestões.

\section{Referências Bibliográficas}

ALEIXO, A. 2001. Conservação da avifauna da Floresta Atlântica: efeitos da fragmentação e a importância de florestas secundárias. In Ornitologia e conservação: da ciência às estratégias (J.L.B. Albuquerque, J.F. Cândido Junior, F.C. Straube \& A.L. Roos, ed.). Unisul, Tubarão, p.199-206.

ALEIXO, A. \& GALETTI, M. 1997. The conservation of the avifauna in a lowland Atlantic Forest in southeast Brazil. Bird Conserv. Int. 7(3):235261. http://dx.doi.org/10.1017/S0959270900001556

ANTUNES, A.Z., ESTON, M.R., SANTOS, A.M.R. \& MENEZES, G.V. 2006. Avaliação das informações disponíveis sobre a avifauna do Parque Estadual Carlos Botelho. Rev. Inst. Flor. 18:103-120.

Antunes, A.Z., Alvarenga, H., SilveirA, L.F., ESTON, M.R., MENEZES, G.V. \& SANTOS, A.S.R. 2007. Distribuição de Leptasthenura setaria (Temminck, 1824) (Aves: Furnariidae) no Estado de São Paulo (Short Communication). Biota Neotrop. 7(1): http:// www.biotaneotropica.org.br/v7n1/pt/abstract?shortcommunication+ bn00607012007.

ANTUNES, A.Z., ESTON, M.R., SILVA, B.G. \& SANTOS, A.M.R. 2011. Comparação entre as comunidades de aves de duas fitofisionomias florestais contíguas no Parque Estadual Carlos Botelho, SP. Neotrop. Biol. Conserv. 6(3):213-226.

AYRES, M., AYRES JUNIOR, M., AYRES, D.L. \& SANTOS, A.S. 2008. BioEstat 5.0 Aplicações estatísticas nas áreas das ciências biológicas e médicas. Instituto de Desenvolvimento Sustentável Mamirauá, Belém.

BARlOW, J., GARDNER, T.A., LOUZADA, J. \& PERES, C.A. 2010. Measuring the conservation value of tropical primary forests: the effect of occasional species on estimates of biodiversity uniqueness. PLoS ONE 5(3):e9609. http://dx.doi.org/10.1371/journal.pone.0009609 
BEISIEGEL, B.M. 2006. Shelter availability and use by mammals and birds in na Atlantic forest área. Biota Neotrop. 6(1): http://www.biotaneotropica. org.br/v6n1/pt/abstract?article+bn00206012006.

BEISIEGEL, B.M. 2007. Foraging association between coatis (Nasua nasua) and birds of the Atlantic forest, Brazil. Biotropica 39(2):383-385. http:// dx.doi.org/10.1111/j.1744-7429.2006.00255.x

BEISIEGEL, B.M. 2009. First camera trap record of bush dogs in the state of São Paulo, Brazil. Canid news 12:5.

BENCKE, G.A., MAURÍCIO, G.N., DEVELEY, P.F. \& GOERCK, J.M. 2006. Áreas Importantes para a Conservação das Aves no Brasil. Parte I - Estados do Domínio da Mata Atlântica. SAVE Brasil, São Paulo.

BERNARDO, C.S.S., RUBIM, P., BUENO, R.S., BEGOTTII, R.A., MEIRELLES, F., DONATTI, C.I., DENZIN, C., STEFFLER, C.E., MARQUES, R.M., BOVENDORP, R.S., GOBBO, S.K. \& GALETTI, M. 2011. Density Estimates of the Black-Fronted Piping Guan in the Brazilian Atlantic Rainforest. Wilson J. Ornithol. 123(4):690-698. http:// dx.doi.org/10.1676/10-140.1

BIBBY, C.J., BURGESS, N.D. \& HILL, D.A. 1992. Bird census techniques. Academic Press, Orlando.

BRASIL. Governo Federal. 2000. SNUC - Sistema Nacional de Unidades de Conservação: Lei ${ }^{\circ}$ 9.985, de 18 de julho de 2000, regulamenta o artigo 225 da Constituição Federal e institui o Sistema Nacional de Unidades de Conservação e da outras providências. Diário Oficial da República Federativa do Brasil, Brasília, DF, 19 jul. 2000.

BRASIL. Ministério do Meio Ambiente. 2007. Áreas Prioritárias para Conservação, Uso Sustentável e Repartição de Benefícios da Biodiversidade Brasileira. Secretaria de Biodiversidade e Florestas, Brasília.

BRoCARDO, C.R., RODARTE, R., BUENO, R.S., CULOT, L. \& GALETTI, M. 2012. Non-volant mammals of Carlos Botelho State Park, Paranapiacaba Forest Continuum. Biota Neotrop. 12(4): http://www. biotaneotropica.org.br/v12n4/pt/abstract?inventory+bn02512042012

CABANNE, G.S., ZURITA, G.A., SEIPKE, S.H. \& BELLOCQ, M.I. 2007. Range expansion, density and conservation of the araucaria Tit-spinetail Leptasthenura setaria (Furnariidae) in Argentina: the role of araucaria Araucaria angustifolia (Araucariaceae) plantations. Bird Cons. Int. 17(4):341-349.

CASTRO, E.R., CORRÊA, M.C., NAVARRO, L., GALETTI, M. \& MORELLATO, L.P.C. 2012. Temporal variation in the abundance of two species of thrushes in relation to fruiting phenology in the Atlantic rainforest. Emu 112(1):137-148. http://dx.doi.org/10.1071/MU11023

CAVARZERE, V., MORAES, G.P. \& SILVEIRA, L.F. 2010. Boracéia Biological Station: an ornithological review. Pap. Avulsos Zool. 50(13): 189-201. http://dx.doi.org/10.1590/S0031-10492010001300001

COHN-HAFT, M., WHITTAKER, A. \& STOUFFER, P.C. 1997. A new look at the "species-poor" Central Amazon: the avifauna North of Manaus, Brazil. Ornithol. Monogr. 48:205-235. http://dx.doi.org/10.2307/40157535

COLWELL, R.K. 2009. EstimateS: Statistical estimation of species richness and shared species from samples. http://purl.oclc.org/estimates (último acesso em 18/01/2011).

COMITÊ BRASILEIRO DE REGISTROS ORNITOLÓGICOS. 2011. Listas das aves do Brasil. 10. ed. versão 25/01/2011. http://www.cbro.org.br (último acesso em 28/05/2011).

FIGUEIREDO, L.F.A. \& CUSTÓDIO-FILHO, A. 2003. Aves observadas em unidades de conservação do estado de São Paulo por Antonio Flávio Barbosa. Bol. CEO. 15:30-43.

FRANCISCO, M.R., OLIVEIRA JUNIOR, P.R.R. \& LUNARDI, V.O. 2008 Nest and fledglings of the Red-ruffed Fruitcrow (Pyroderus scutatus). Wilson J. Ornithol. 120(2):413-416. http://dx.doi.org/10.1676/07-030.1

GALETTI, M. \& ALEIXO, A. 1997. Effects of palm heart harvesting on avian frugívoras in the Atlantic rain forest of Brazil. J. Applied Ecol. 35(2):286293. http://dx.doi.org/10.1046/j.1365-2664.1998.00294.x
GAletTI, M., MARTUSCELli, P., OLMOS, F. \& ALEIXO, A. 1997. Ecology and conservation of the jacutinga Pipile jacutinga in the Atlantic forest of Brazil. Biol. Conserv. 82(1):31-39. http://dx.doi.org/10.1016/ S0006-3207(97)00004-9

GALETTI, M., GIACOMINI, H.C., BUENO, R.S., BERNARDO, C.S.S., MARQUES, R.M., BOVENDORP, R.S., STEFFLER, C.E., RUBIM, P., GOBBO, S.K., DONATTI, C.I., BEGOTTI, R.A., MEIRELLES, F., NOBRE, R.A., CHIARELlO, A.G. \& PERES, C.A. 2009. Priority areas for the conservation of Atlantic Forest large mammals. Biol. Conserv. 142(6):1229-1241. http://dx.doi.org/10.1016/j. biocon.2009.01.023

GUIX, J.C., MARTÍN, M.C. \& SERRA, A. 2002. Density estimates of five syntopic species of parrots (Aves: Psittacidae): population status in the Paranapiacaba fragment. In Censuses of vertebrates in a Brazilian Atlantic rainforest area: the Paranapiacaba fragment (E. Mateos, J.C. Guix, A. Serra \& K. Pisciotta, eds.). Universitat de Barcelona, Barcelona, p.95-110.

HARRIS, G.M. \& PIMM, S.L. 2004. Bird species' tolerance of secondary forest habitats and its effects on extinction. Conserv. Biol. 18(6):16071616

HERNÁNDEZ, A., MARTÍN, M., SERRA, A. \& GUIX, J.C. 2002. Density estimates of syntopic species of toucans (Aves: Ramphastidae). In Censuses of vertebrates in a Brazilian Atlantic rainforest area: the Paranapiacaba fragment (E. Mateos, J.C. Guix, A. Serra \& K. Pisciotta, eds.). Universitat de Barcelona, Barcelona, p.79-94.

INSTITUTO BRASILEIRO DE GEOGRAFIAE ESTATÍSTICA-IBGE. 2012 Manual técnico da vegetação brasileira. 2. ed. IBGE, Rio de Janeiro.

MAGALHÃES, J.C.R. 1999. As aves na fazenda Barreiro Rico. Plêiade, São Paulo.

MITTERMEIER, R.A., ROBLES, G.P., HOFFMANN, M., PILGRIM, J., BROOKS, T.M., MITTERMEIER, C.G., LAMOREUX, J. \& FONSECA, G.A.B. 2004. Hotspots revisited. CEMEX, Cidade do México.

OLIVEIRA, P.R.R., ALBERTS, C.C. \& FRANCISCO, M.R. 2011. Impact of road clearings on the movements of three understory insectivorous bird species in the Brazilian Atlantic Forest. Biotropica 43(5):628-632. http:// dx.doi.org/10.1111/j.1744-7429.2010.00744.x

PACAGNELLA, S.G., ANTONELLI-FILHO, R., LARA, A.I. \& SCHERERNETO, P. 1994. Observações sobre Pipile jacutinga Spix, 1825 (Aves, Cracidae) no Parque Estadual de Carlos Botelho, São Paulo, Brasil. Iheringia Zool. 76(1):29-32.

PACHECO, J.F. \& FONSECA, P.S.M. 2002. Resultados de excursão ornitológica a determinadas áreas dos estados de São Paulo, Santa Catarina e Rio Grande do Sul em janeiro, 1990. Atual. Ornitol. 106(1):3-5.

PEDROCCHI, V., SILVA, C.R. \& SILVA, A. 2002. Check list of birds and mammals in the Paranapiacaba forest fragment. In Censuses of vertebrates in a Brazilian Atlantic rainforest area: the Paranapiacaba fragment (E. Mateos, J.C. Guix, A. Serra \& K. Pisciotta, eds.). Universitat de Barcelona, Barcelona, p.183-204.

REMSEN, J.V.Jr. 1994. Use and misuse of bird lists in community ecology and conservation. Auk 111(1):225-227. http://dx.doi.org/10.2307/4088531

RIBEIRO, M.C., MARTENSEN, A.C., METZGER, J.P., TABARELLI, M., SCARANO, F. \& FORTIN, M.J. 2011. The Brazilian Atlantic Forest: a shrinking biodiversity hotspot. In Biodiversity Hotspots: distribution and protection of conservation priority areas (F.E. Zachos \& J.C. Habel, eds.). Springer-Verlag, London, p.405-434.

ROCCA, M.A. \& SAZIMA, M. 2008. Ornithophilous canopy species in the Atlantic Rain Forest of southeastern Brazil. J. Field Ornithol. 79(2):130137. http://dx.doi.org/10.1111/j.1557-9263.2008.00154.x

ROCCA, M.A., SAZIMA, M. \& SAZIMA, I. 2006. Woody woodpecker enjoys soft drinks: the blond-crested woodpecker seeks néctar and pollinates canopy plants in southeastern Brazil. Biota Neotrop. 6(2): http://www.biotaneotropica.org.br/v6n2/pt/abstract?shortcommunicatio n+bn026066022006

RODRIGUES, R.R. \& BONONI, V.L.R. (Org.). 2008. Diretrizes para a conservação e recuperação da Biodiversidade no Estado de São Paulo. Imprensa Oficial, São Paulo. 
SÁNCHEZ-ALONSO, C., OLIVERAS, I. \& MARTÍN, M. 2002. Density estimates of guans (Aves: Cracidae): Pipile jacutinga and Penelope obscura. In Censuses of vertebrates in a Brazilian Atlantic rainforest area: the Paranapiacaba fragment (E. Mateos, J.C. Guix, A. Serra \& K. Pisciotta, eds.). Universitat de Barcelona, Barcelona, p. 67-78.

SÃO PAULO (Estado). 2008a. Plano de Manejo do Parque Estadual Carlos Botelho. http://www.fflorestal.sp.gov.br/planodemanejoCompletos.php (último acesso em 20/01/2011).

SÃO PAULO (Estado). 2008b. Decreto n ${ }^{\circ} 53.494$, de 2 de outubro de 2008. Declara as espécies da fauna silvestre ameaçadas, as quase ameaçadas e com dados insuficientes para avaliação no Estado de São Paulo e dá providências correlatas. Diário Oficial do Estado de São Paulo, Poder Executivo, São Paulo, 3 out. 2008. Seção I, n.118 (187), p.2-10.

SICK, H. 1997. Ornitologia brasileira. Nova Fronteira, Rio de Janeiro.

SILVA, B.G., ANTUNES, A.Z. \& ESTON, M.R. 2010. Variação local na composição da comunidade de aves no Parque Estadual Carlos Botelho, São Miguel Arcanjo-SP e propostas para o monitoramento do impacto do ecoturismo. Rev. Inst. Florest. 22(2):215-232.
SILVEIRA, L.F. \& UEZU, A. 2011. Checklist of birds from São Paulo State, Brazil. Biota Neotrop. 11(1a): http://www.biotaneotropica.org.br/v11n1a/ en/abstract?inventory+bn0061101a2011.

STRAUBE, F.C. \& SCHERER-NETO, P. 1995. Novas observações sobre a "cunhataí" Triclaria malachitacea (Spix, 1824) nos Estados do Paraná e São Paulo (Psittacidae, Aves). Acta Biol. Leopold. 17(1):147-152.

TERBORGH, J. \& VAN SCHAIK, C. 2002. Por que o mundo necessita de parques. In Tornando os parques eficientes: estratégias para a conservação da natureza nos trópicos (J. Terborgh, C. Van Schaik, L. Davenport \& M. Rao, orgs.). Editora da UFPR e Fundação O Boticário, Curitiba, p.25-36.

VIELLIARD, J.M.E. \& SILVA, W.R. 2001. Avifauna. In: Intervales (Secretaria de Estado do Meio Ambiente, org.). Imprensa Oficial, São Paulo, p.124-145.

WILLIS, E.O. \& ONIKI, Y. 1981. Levantamento preliminar de aves em treze áreas do Estado de São Paulo. Rev. Brasil. Biol. 41(1): 121-135.

WILLIS, E.O. \& ONIKI, Y. 2002. Birds of Santa Teresa, Espírito Santo, Brazil: Do humans add or subtract species? Papéis Avulsos de Zool. 42(9):193264. http://dx.doi.org/10.1590/S0031-10492002000900001

WILLIS, E.O. \& ONIKI, Y. 2003. Aves do Estado de São Paulo. Divisa, Rio Claro. 